\title{
Quantum Brownian motion with inhomogeneous damping and diffusion
}

\author{
Pietro Massignan, ${ }^{1}$ Aniello Lampo, ${ }^{1} \mathrm{Jan}$ Wehr, ${ }^{2}$ and Maciej Lewenstein ${ }^{1,3, *}$ \\ ${ }^{1}$ ICFO-Institut de Ciències Fotòniques, Av. C.F. Gauss, 3, E-08860 Castelldefels, Spain \\ ${ }^{2}$ Department of Mathematics, University of Arizona, Tucson, Arizona 85721-0089, USA \\ ${ }^{3}$ ICREA-Institució Catalana de Recerca i Estudis Avançats, Lluis Companys 23, E-08010 Barcelona, Spain
}

(Received 10 November 2014; published 23 March 2015)

\begin{abstract}
We analyze the microscopic model of quantum Brownian motion, describing a Brownian particle interacting with a bosonic bath through a coupling which is linear in the creation and annihilation operators of the bath, but may be a nonlinear function of the position of the particle. Physically, this corresponds to a configuration in which damping and diffusion are spatially inhomogeneous. We derive systematically the quantum master equation for the Brownian particle in the Born-Markov approximation and we discuss the appearance of additional terms, for various polynomials forms of the coupling. We discuss the cases of linear and quadratic coupling in great detail and we derive, using Wigner function techniques, the stationary solutions of the master equation for a Brownian particle in a harmonic trapping potential. We predict quite generally Gaussian stationary states, and we compute the aspect ratio and the spread of the distributions. In particular, we find that these solutions may be squeezed (superlocalized) with respect to the position of the Brownian particle. We analyze various restrictions to the validity of our theory posed by non-Markovian effects and by the Heisenberg principle. We further study the dynamical stability of the system, by applying a Gaussian approximation to the time-dependent Wigner function, and we compute the decoherence rates of coherent quantum superpositions in position space. Finally, we propose a possible experimental realization of the physics discussed here, by considering an impurity particle embedded in a degenerate quantum gas.
\end{abstract}

DOI: 10.1103/PhysRevA.91.033627

PACS number(s): 03.75.Gg, 05.40.-a, 03.65.Yz, 72.70.+m

\section{INTRODUCTION}

The theory of quantum Brownian motion (QBM) has been a subject of studies for decades and belongs nowadays to a standard textbook material [1-5]. Nevertheless, there are some aspects of QBM that have not been, in our opinion, explored completely in the literature, and that is what motivates our paper.

First, one should note that the vast majority of the work on QBM is devoted to microscopic models in which the coupling of the Brownian particle to the bosonic bath is linear both in bath creation and annihilation operators, and in position (or momentum) of the particle. The case when such coupling is nonlinear in either the bath or the system operators has been hardly studied; unique exceptions to our knowledge provide the old works of Landauer [6], who studied nonlinearity in bath operators, and Dykman and Krivoglaz [7], Hu, Paz, and Zhang [8], Brun [9], and Banerjee and Ghosh [10], who considered both cases. Physically, the case of a coupling which deviates from linearity in the system coordinates corresponds to a situation in which damping and diffusion are spatially inhomogeneous. Obviously, such nonlinearity might have both classical and quantum consequences, and as such deserves careful analysis.

Second, this type of inhomogeneity has been recently intensively studied in the context of classical Brownian motion (CBM) and other classical diffusive systems. In particular, explicit formulas were derived for noise-induced drifts in the small-mass (Smoluchowski-Kramers [11,12]) and other limits [13-15]. Noise-induced drifts have been shown to appear in

\footnotetext{
"maciej.lewenstein@icfo.es
}

a general class of diffusive systems, including systems with time delay and systems driven by colored noise. Applications include Brownian motion in diffusion gradient $[16,17]$, noisy electrical circuits [18], and thermophoresis [19]. In the first two cases, the theoretical predictions have been demonstrated to be in an excellent agreement with the experiment. Diffusion in inhomogeneous and disordered media is presently one of the fastest developing subjects in the theory of random walks and CBM [20-23], and finds vast applications in various areas of science. There is a considerable interest in the studies of various forms of anomalous diffusion and nonergodicity [23-26], based either on the theory of heavy-tailed continuoustime random walk (CTRW) $[27,28]$ or on models characterized by a diffusivity (i.e., a diffusion coefficient) that is inhomogeneous in time [29] or space [13,30]. Particularly impressive is the recent progress in single-particle imaging, for instance in biophotonics (cf. [31-37] and references therein), where the single-particle trajectories of, say, a receptor on a cell membrane can be traced. It is presently investigated how random walk and CBM models with inhomogeneous diffusion may be employed in the description of such phenomena $[38,39]$.

The examples mentioned above are strictly classical, but the recent unprecedented progress in control, detection, and manipulation of ultracold atoms and ions [40] are giving us the possibility to perform similar kind of experiments (e.g., singleparticle tracking to monitor the real-time dynamics of given atoms) in the quantum regime [41]. Note that such experiments were unthinkable, say, 20 years ago (see the corresponding paragraphs about difficulties to observe QBM in Ref. [1]). Note also that ultracold setups will naturally involve spatial inhomogeneities, due to the necessary presence of trapping potentials and eventual stray fields. This is in fact the third motivation of this paper: to formulate and study the theory 
of QBM at low temperatures, and in the presence of spatially inhomogeneous damping and diffusion.

An immediate application of our theory concerns dilute impurities embedded in an ultracold degenerate quantum gas. Such problem has been intensively studied in the recent years in the context of polaron physics in strongly interacting Fermi gases [42-47] and Bose gases [48-57]. Obviously, there is a vast amount of literature on the polaron problem, or more generally on electron-phonon interactions, in solid-state systems (cf. $[58,59])$. The theory of polarons has been also a subject of intensive studies in mathematical physics [60-63]. In analogy to the studies of classical stochastic processes [13,14,64-68], this work opens also the possibility of employing ultracold atoms to study the quantum Smoluchowski-Kramers limit of a very light Brownian particle, or correspondingly an overdamped Brownian motion.

Since this paper revisits some of the handbook material, part of the presentation reproduces known and well-established results. We include it here in order to make our further argumentations and derivations self-contained. We start in Sec. II by presenting the microscopic model of QBM, known as Caldeira-Leggett model [69,70], and we derive the quantum master equation (QME) in the Born-Markov approximation, following up to a certain point the standard weak-coupling treatment, i.e., by means of perturbation theory to second order in the bath-system coupling constant [1]. The resulting equation is systematic in the sense of Born expansion, and it takes a certain part of non-Markovian effects into account. In its most common form, the QME is derived in the limit when the characteristic energy of the system (i.e., the Brownian particle) $\hbar \Omega$ is much smaller than the cutoff energy $\hbar \Lambda$, and the latter is much smaller than the thermal energy $k_{B} T$ of the bath; in the following, we will refer to this regime as the Caldeira-Leggett limit. However, in this paper we are interested to the regime where $k_{B} T$ becomes comparable to $\hbar \Omega$. Section III deals with the case of linear coupling, i.e., spatially homogeneous damping and diffusion; although this case has been widely elaborated previously [2,3], we discuss carefully the nonstandard modifications of the generalized master equation appearing in the uncommon limit $\hbar \Lambda \gg$ $\hbar \Omega \sim k_{B} T$. In Sec. IV, we present our results concerning a coupling which is quadratic in the position of the test particle, which yields a quadratic dependence of the damping and diffusion coefficients on the position of the Brownian particle, and extract the corresponding position-space decoherence time. The stationary solutions of the QMEs and their properties for linear and quadratic coupling are discussed in Sec. V. We predict quite generally Gaussian stationary states which are asymmetric in the position and momentum variables, and that may be classified in terms of an effective cooling or heating, depending on whether the associated distribution is more or less spread out than the one of its quantum thermal Gibbs-Boltzmann counterpart. The aspect ratio of the distribution can be so extreme that the system may even become squeezed (superlocalized) with respect to the position of the Brownian particle. The squeezing effect can be understood in terms of renormalization, or Lamb shift, of the system frequency $\hbar \Omega$ due to virtual excitations by the nonresonant bath modes. We analyze various restrictions on the validity of our theory imposed by Heisenberg principle and
non-Markovian effects, and we stress the role and possibility of observation of quantum effects. In Sec. VI, we discuss the near-equilibrium dynamics of the system by computing moments of the time-dependent Wigner function. We conclude and present the outlook in Sec. VII, where we comment on the experimental realization of the models described by our theory. There, we also comment on challenges of investigating the so-called Smoluchowski-Kramers limit using a quantum analog of classical homogenization theory (cf. [14]). A number of more intricate issues are addressed in the Appendices. In Appendix A, we discuss the most general QME for the case of a generical polynomial coupling in the system's position, and Appendices B and C deal with a rather technical point, the detailed calculation of the coefficients appearing in the generic QME. In Appendix D, we summarize the asymptotic behavior of the QME coefficients for the cases of a linear and quadratic coupling. In Appendix E, we analyze a (somehow oversimplified) high-temperature limit of the QME, which includes however the leading quantum corrections. Finally, Appendix F discusses challenges related to application of our theory to the problem of an impurity in an ultracold quantum gas.

It is important to stress to which extent our paper goes beyond the results of the previously published work [8-10]. In particular, the in-depth study of $\mathrm{Hu}, \mathrm{Paz}$, and Zhang contains the derivations of time-dependent (Redfield) and time-independent master equation for the case of general system-bath coupling: linear or nonlinear in bath and system operators. In our paper, we consider the case where the coupling is linear in bath operators and polynomial in the system position $x$, but in contrast to the earlier works we provide the following: (i) a careful analysis of the parameter dependencies of coefficients entering into the timeindependent master equation, obtained as a long-time limit of the Redfield equation, and the various limits of the resulting equation; (ii) a derivation and a detailed discussion of the properties of the stationary solutions, analyzing in particular their dynamical stability, classifying solutions in terms of an effective cooling or heating, and highlighting the presence of quantum squeezed regimes; (iii) a discussion of QBM in the context of physics of ultracold degenerate gases; in particular, this paper provides a solid theoretical basis for further studies of quantum Brownian motion of an impurity atom inside a Bose-Einstein condensate.

Before turning to the body of the paper, let us clarify the use of the notions nonlinear or nonlinear coupling we will use in the following. In this paper, we limit ourselves to bosonic baths with effective bath Hamiltonians which are quadratic in bath creation and annihilation operators (i.e., the bath dynamics is linear here), although more general cases with bath Hamiltonians containing also quartic terms are also of physical interest (i.e., the bath dynamics is itself nonlinear there; cf. [55]). We limit ourselves also to system Hamiltonians quadratic in the system's position and momentum (i.e., the system dynamics is also linear here). Obviously, nonharmonic trapping or spatially periodic or even random potentials (leading to a nonlinear dynamics for the system itself) are also of physical interest. When we refer in this paper to linear coupling between the system and the bath, we consider the situation when the coupling between the bath and the system 
has the following form: a bath operator linear in the bath creation and annihilation operator times a system operator linear in the system's particle position, or momentum, or both. Note that if in such situation both the Hamiltonian of the bath and of the system are quadratic, the whole model corresponds to a system of coupled harmonic oscillators (i.e., the system plus bath dynamics as a whole is linear), and the model is exactly solvable by standard methods (via, e.g., matrix diagonalization or Fourier or Laplace techniques). We refer to concrete examples in the following. When instead we consider a nonlinear coupling between the system and the bath, we refer to the situation when the coupling between the bath and the system has the following form: a bath operator linear in the bath creation and annihilation operator times a system operator nonlinear in the system's particle position, or momentum, or both. Of course, one may also consider a nonlinear coupling between a bath and a system which are themselves nonlinear (cf. [8]). To conclude, the nonlinearity in the coupling (which is the main focus of this work) should not be confused with the nonlinear order of the Born expansion in the coupling constant $\kappa_{k}$. Models involving expansions to quartic and higher order in $\kappa_{k}$ have been discussed in detail elsewhere (see, e.g., [71] and references therein).

\section{CALDEIRA-LEGGETT MODEL AND QUANTUM MASTER EQUATION}

\section{A. Caldeira-Leggett model}

The Caldeira-Leggett model (CLM) is one of many models describing a (Brownian) particle interacting with a bosonic bath (for the models discussing interaction of an atom, or ensemble of atoms, with a minimally coupled photon bath, see for instance [72,73]). Despite its simplicity, the CLM gained popularity in condensed matter physics due to its very general nature, and its ability to describe quantum dissipation in the Ohmic, super-Ohmic, and sub-Ohmic limits. The model is defined by the Hamiltonian

$$
H=H_{\mathrm{S}}+H_{\mathrm{B}}+H_{\mathrm{I}},
$$

where the system, bath, and interaction Hamiltonians are, respectively,

$$
\begin{gathered}
H_{\mathrm{S}}=H_{\mathrm{sys}}+V_{c}(x)=\frac{p^{2}}{2 m}+V(x)+V_{c}(x), \\
H_{\mathrm{B}}=\sum_{k}\left(\frac{p_{k}^{2}}{2 m_{k}}+\frac{m_{k} \omega_{k}^{2} x_{k}^{2}}{2}\right)-E_{0}=\sum_{k} \hbar \omega_{k} g_{k}^{\dagger} g_{k}, \\
H_{\mathrm{I}}=-f(x) B=-\sum_{k} \kappa_{k} x_{k} f(x) .
\end{gathered}
$$

In the above expressions, $p$ is the particle momentum, $m$ its mass, $V(x)$ the trapping potential, and the so-called counterterm

$$
V_{c}(x)=\sum_{k} \frac{\kappa_{k}^{2}}{2 m_{k} \omega_{k}^{2}} f(x)^{2}
$$

will be needed in the following to remove unphysical divergent renormalizations of the trapping potential arising from the coupling to the bath. The bath bosons have masses $m_{k}$ and frequencies $\omega_{k}$, and their momenta and position are denoted by $p_{k}$ and $x_{k}$, respectively. Alternatively, we describe them with the help of annihilation and creation operators $g_{k}$ and $g_{k}^{\dagger}$. From the bath Hamiltonian, we have removed the constant zero-point energy $E_{0}$. The parameters describing the coupling of the bath modes to the system are denoted by $\kappa_{k}$. We consider here the case of a very general position-dependent coupling, described by a function $f(x)$ of the particle position $x$. To keep notation as close as possible to the usual case of linear coupling, we take $f(x)$ to have dimension of length, i.e., we write it as $f(x)=a \tilde{f}(x / a)$, with $\tilde{f}(x)$ being dimensionless and $a$ denoting a typical length scale on which $f$ varies. We will restrict our discussion in the following to the one-dimensional (1D) case, but generalizations to $2 \mathrm{D}$ or $3 \mathrm{D}$ are straightforward.

Since in order to derive the QME we are going to use systematic Born-Markov approximation, it is useful to identify orders of magnitude of various terms with respect to the coupling. To this aim, we rewrite the Hamiltonian as

$$
H=H_{0}+H_{1}+H_{2},
$$

where $H_{0}=H_{\text {sys }}+H_{B}, H_{1}=H_{\mathrm{I}}$, and $H_{2}=V_{c}(x)$. The Hamiltonian of the system+bath ensemble may be written as

$$
H=H_{\text {sys }}+V_{c}(x)+\sum_{k} \hbar \omega_{k} g_{k}^{\dagger} g_{k}+\sqrt{\frac{\hbar \kappa_{k}^{2}}{2 m_{k} \omega_{k}}}\left(g_{k}+g_{k}^{\dagger}\right) f(x) \text {. }
$$

The next steps consist in going to the interaction picture with respect to $H_{0}$, writing the Liouville-von Neumann equation for the total density matrix $\rho(t)$ of the system and bath

$$
\dot{\rho}(t)=-\frac{i}{\hbar}\left[H_{\mathrm{I}}(t), \rho\right],
$$

where $H_{\mathrm{I}}(t)$ is the interaction Hamiltonian in the interaction picture. We solve the above equation formally

$$
\rho(t)=\rho(0)-\frac{i}{\hbar} \int_{0}^{t} d s\left[H_{\mathrm{I}}(s), \rho(s)\right],
$$

and insert the solution into (8). Taking trace over the bath and assuming $^{1}$ that $\operatorname{tr}_{\mathrm{B}}\left[H_{\mathrm{I}}(t), \rho(0)\right]=0$ we obtain

$$
\dot{\rho}_{S}(t)=-\frac{1}{\hbar^{2}} \int_{0}^{t} d s \operatorname{Tr}_{B}\left[H_{\mathrm{I}}(t),\left[H_{\mathrm{I}}(s), \rho(s)\right]\right] .
$$

\section{B. Born-Markov approximation}

We assume also that initially the system and the bath were uncorrelated, i.e., the initial density matrix was a simple tensor product $\rho_{S}(0) \otimes \rho_{B}(0)$. The first approximation that we apply is the Born approximation: in a weak-coupling regime, we expect that the influence of the system on the

\footnotetext{
${ }^{1}$ This assumption is typically verified as a consequence of the symmetries; the initial state $\rho(0)$ is often taken to be an even function of the bath modes' position and momentum operators, while the interaction Hamiltonian is an odd function. In any case, this condition may always be satisfied by suitably redefining the Hamiltonian.
} 
bath is negligible, and the state of the total system remains approximately uncorrelated for all times

$$
\rho(t) \simeq \rho_{S}(t) \otimes \rho_{B}(0) .
$$

Under this standard approximation (cf. [2]), we obtain first

$$
\dot{\rho}_{S}(t)=-\frac{1}{\hbar^{2}} \int_{0}^{t} d s \operatorname{Tr}_{B}\left[H_{\mathrm{I}}(t),\left[H_{\mathrm{I}}(s), \rho_{S}(s) \otimes \rho_{B}(0)\right]\right] .
$$

The next steps require more specific assumptions about the initial state of the bath, and an explicit form of the bath parameters $\kappa_{k}, m_{k}$, and $\omega_{k}$. We will assume a thermal state of the bath, described by the density matrix

$$
\rho_{B}(0)=\frac{\exp \left(-H_{B} / k_{B} T\right)}{\operatorname{Tr}_{B}\left[\exp \left(-H_{B} / k_{B} T\right)\right]} .
$$

We will also introduce the spectral density, which contains all the relevant properties of the bath; it determines the analytical form of the coefficients of the QME, and therefore characterizes the main dissipation and decoherence processes occurring in the central system. The spectral density may be generally defined as

$$
J(\omega)=\sum_{k} \frac{\kappa_{k}^{2}}{2 m_{k} \omega_{k}} \delta\left(\omega-\omega_{k}\right) .
$$

As we will see in the following [see Eq. (25)], the spectral density will be more specifically defined to be proportional to a damping constant $\gamma$, and will necessarily contain a UV momentum cutoff $\Lambda$. As such, when taking the trace over the bath degrees of freedom, the bath correlation functions arising in Eq. (12) will decay on a fast characteristic time scale $\tau_{B}$, determined by $1 / \Lambda$ and $\hbar / k_{B} T$. On the other hand, in presence of a weak coupling between the bath and the system, the interaction-picture system density matrix $\rho_{S}(t)$ will evolve only on a much slower time scale, set by $1 / \gamma$. We may thus safely shift $\rho_{S}(s)$ to $\rho_{S}(t)$ in Eq. (12). Note that even if the system exhibits at long times algebraic decay of the form $C / t^{v}$ with some exponent $v$ of order 1 , the shift from $s$ to $t$ for $|t-s|<\tau_{B}$ causes a relative error of order $\nu \tau_{B} / t$, which is negligible at long times. Traditionally, this approximation is termed in the handbooks [1-5] Markov approximation, although the considered quantum stochastic process strictly speaking is non-Markovian. In the following, we will see this approximation actually is part of the systematic second-order (weak-coupling) expansion in the coupling constant: the shift from $s$ to $t$ will be accompanied by the corresponding zerothorder time translation (i.e., time translation for the system decoupled from the bath).

In this way, we derive the so-called Redfield equation $[74,75]$ for the reduced density matrix of the systems. Going back to the Schrödinger picture, the latter reads as

$$
\begin{aligned}
\dot{\rho}_{S}(t)= & -\frac{i}{\hbar}\left[H_{S}, \rho_{S}\right]-\frac{1}{\hbar^{2}} \int_{0}^{t} d \tau \operatorname{Tr}_{B}\left[H_{\mathrm{I}}(0),\right. \\
& {\left.\left[H_{\mathrm{I}}(-\tau), \rho_{S}(t) \otimes \rho_{B}(0)\right]\right] . }
\end{aligned}
$$

Note that the Redfield equation is in fact the systematically derived master equation in the second order of the expansion in coupling constant, also known as weak-coupling master equation. It is explicitly time dependent, and as such it is capable of describing non-Markovian effects. This is discussed in some detail for the case of linear couplings in Ref. [3], and for the general nonlinear couplings in Ref. [8]. To be more specific, the Redfield equation has a well-defined long-time limit, expected to describe correctly the long-time behavior, but it also describes the short-time non-Markovian effects. In many cases, these non-Markovian effects reduce to "initial slips," i.e., rapid changes of the system density matrix before entering into the long-time regime, and an "adiabatic drag," when the systems during the slow, long-time phase of the evolution "drags" the bath with itself (cf. [76-78], and references therein) The final step of what is traditionally called Markov approximation consists in taking the long-time limit, extending the $\tau$ integration to infinity, obtaining in this way a QME which is local in time:

$$
\begin{gathered}
\dot{\rho}_{S}(t)=-\frac{i}{\hbar}\left[H_{S}, \rho_{S}\right]-\frac{1}{\hbar^{2}} \int_{0}^{\infty} d \tau \operatorname{Tr}_{B}\left[H_{\mathrm{I}}(0),\right. \\
\left.\left[H_{\mathrm{I}}(-\tau), \rho_{S}(t) \otimes \rho_{B}(0)\right]\right] .
\end{gathered}
$$

We will refer to the latter as to the Born-Markov quantum master equation (BM-QME), making explicit reference to the two key approximations performed to derive it. At this point, two important issues are worth discussing. First of all, it should be noted that the BM-QME is not, strictly speaking, Markovian. A quantum stochastic process is a quantum Markov process only if it can be regarded as a quantum Langevin process with purely white noise, and if it is described by a time-independent master equation of Lindblad form. If we treated our model solving, say, the Heisenberg equations of motion, we would see the following: (i) for the case of linear coupling, the quantum noise is additive, but by no means white: its correlations indeed would typically have finite (exponential decay) correlation time, and even small algebraic long-time tails; (ii) for the case of nonlinear coupling, the quantum noise not only is colored, but is multiplicative, which of course complicates the treatment even more. Moreover, the long-time limit taken, in Eq. (16), during the Markov approximation, loosely speaking erases the memory about the initial state. Such procedure is a frequent "abuse" in quantum optics, leading to unphysical solutions in certain regimes of parameters (typically at very low temperatures). Obviously, all these problems can be avoided in the case of linear coupling and harmonic trapping potential, when the exact QME is used $[79,80]$. Unfortunately, the exact solutions are not known in the case of nonlinear coupling. In the latter case, the Markov-Born approximations quite naturally seem to be the method of choice to obtain additional results. Trying to improve them using a canonical perturbation theory in the manner of Ref. [71] is a very interesting challenge, which however goes beyond the scope of this paper. In order to obtain a fair comparison, we will compare the approximate solutions of the nonlinear case with the results obtained for the linear coupling using the same Markov-Born approximation.

\section{Caldeira-Leggett QME}

Following the notation of Ref. [3], we can express the environment self-correlation function as $\mathcal{C}(\tau)=\langle B(0) B(-\tau)\rangle_{B}=$ 
$v(\tau)-i \eta(\tau)$, with the noise kernel

$$
\nu(\tau)=\int_{0}^{\infty} d \omega J(\omega) \operatorname{coth}\left(\frac{\hbar \omega}{2 k_{B} T}\right) \cos (\omega \tau)
$$

and the dissipation kernel

$$
\eta(\tau)=\int_{0}^{\infty} d \omega J(\omega) \sin (\omega \tau)
$$

The master equation for the system density matrix $\rho(t)$ (we will skip in the following the subscript $S$ ) takes then the form

$$
\begin{aligned}
\dot{\rho}(t)= & -\frac{i}{\hbar}\left[H_{S}, \rho(t)\right] \\
& -\frac{1}{\hbar^{2}} \int_{0}^{\infty} d \tau(v(\tau)[f(x(0)),[f(x(-\tau)), \rho(t)]] \\
& -i \eta(\tau)[f(x(0)),\{f(x(-\tau)), \rho(t)\}]) .
\end{aligned}
$$

In the case when the coupling is linear in the position of the particle and the environment is Ohmic, Caldeira and Leggett in Refs. [69,70] showed that the reduced density matrix $\rho$ of a harmonic oscillator of mass $m$ and frequency $\Omega$ obeys in the high-temperature limit $k_{B} T / \hbar \gg \Lambda \gg \Omega$ the following master equation (CLME):

$$
\dot{\rho}=-\frac{i}{\hbar}\left[H_{\mathrm{sys}}, \rho\right]-\frac{i \gamma}{2 \hbar}[x,\{p, \rho\}]-\frac{m \gamma k_{B} T}{\hbar^{2}}[x,[x, \rho]] .
$$

Here, $\gamma=\eta / m$ is the characteristic damping rate of the oscillator, and $\eta$ is the friction coefficient.

Similarly, as shown first in Ref. [8], in the case of nonlinear coupling $f(x)$ to the Ohmic environment and $T \rightarrow \infty$, the evolution of the system is described by a generalization of the Caldeira-Leggett master equation, which may be written as

$$
\begin{aligned}
\dot{\rho}= & -\frac{i}{\hbar}\left[H_{\mathrm{sys}}, \rho\right]-\frac{i \gamma m}{2 \hbar}[f(x),\{\dot{f}(x), \rho\}] \\
& -\frac{m \gamma k_{B} T}{\hbar^{2}}[f(x),[f(x), \rho]] .
\end{aligned}
$$

We have introduced and defined here the "dot" operator

$$
\dot{f}(x)=-\frac{i}{\hbar}\left[f(x), H_{\mathrm{sys}}\right]=\frac{p f^{\prime}(x)+f^{\prime}(x) p}{2 m} .
$$

Our aim in the following is to derive the generalizations of Eqs. (20) and (21) to the situation in which $k_{B} T \simeq \hbar \Omega$, and the largest energy scale in the problem is the cutoff energy $\hbar \Lambda$. For the case of linear coupling, the resulting master equation was derived in certain limits in Refs. [2,3]. One should stress once more that in the case when the Hamiltonians of both the bath and the system are quadratic, the whole model corresponds to a system of coupled harmonic oscillators (i.e., the system plus bath dynamics as a whole is linear), and the model is exactly soluble by standard methods such as matrix diagonalization, Fourier or Laplace techniques (cf. [72,81,82]). In such situation, the exact timedependent master equation (i.e., an exact analog of the Redfield equation) can be worked out rigorously for various spectral functions (cf. [79,80]). The case of nonlinear coupling (in the system's variables, as discussed in the last paragraph of the
Introduction), to our knowledge, has been only discussed in Refs. [8-10]; however, explicit analytic expressions for the coefficients entering the master equation have generally not been discussed there.

\section{BM-QME WITH LINEAR COUPLING}

In this work, we will focus on the simplest case of a harmonic potential $V(x)=m \Omega^{2} x^{2} / 2$, where $\Omega$ denotes the oscillator frequency, and $m \Omega^{2}$ is the corresponding spring constant. In the interaction picture, the position operator obeys $x(\tau)=x \cos (\Omega \tau)+(p / m \Omega) \sin (\Omega \tau)$, and the master equation may be written in the simple form

$$
\begin{aligned}
\dot{\rho}(t)= & -\frac{i}{\hbar}\left[\hat{H}_{S}+C_{x} x^{2}, \rho(t)\right]-\frac{i C_{p}}{\hbar m \Omega}[x,\{p, \rho(t)\}] \\
& -\frac{D_{x}}{\hbar}[x,[x, \rho(t)]]-\frac{D_{p}}{\hbar m \Omega}[x,[p, \rho(t)]],
\end{aligned}
$$

where the frequency renormalization of the harmonic potential, the momentum damping coefficient, the normal diffusion coefficient, and the anomalous diffusion coefficient are, respectively, proportional to

$$
\begin{aligned}
& C_{x}=-\int_{0}^{\infty} d \tau \eta(\tau) \cos (\Omega \tau), \\
& C_{p}=\int_{0}^{\infty} d \tau \eta(\tau) \sin (\Omega \tau), \\
& D_{x}=\int_{0}^{\infty} d \tau \nu(\tau) \cos (\Omega \tau), \\
& D_{p}=-\int_{0}^{\infty} d \tau \nu(\tau) \sin (\Omega \tau) .
\end{aligned}
$$

For definiteness, in this paper we focus on the case where the spectral density is Ohmic (i.e., it is linear in $\omega$ ) and has a Lorentz-Drude (LD) cutoff

$$
J(\omega)=\frac{m \gamma}{\pi} \omega \frac{\Lambda^{2}}{\omega^{2}+\Lambda^{2}} .
$$

The specific choice of cutoff function yields minor quantitative changes to the QME coefficients, but as physically expected, it does not alter their asymptotic behavior. Exploiting the Matsubara representation

$$
\operatorname{coth}\left(\frac{\hbar \omega}{2 k_{B} T}\right)=\frac{2 k_{B} T}{\hbar \omega} \sum_{n=-\infty}^{\infty} \frac{1}{1+\left(v_{n} / \omega\right)^{2}}
$$

with bosonic frequencies $v_{n}=2 \pi n k_{B} T / \hbar$, the noise and dissipation kernels may be evaluated analytically with the help of the Cauchy's residue theorem

$$
\begin{gathered}
\nu(\tau)=\frac{m k_{B} T \gamma \Lambda^{2}}{\hbar} \sum_{n=-\infty}^{\infty} \frac{\Lambda e^{-\Lambda|\tau|}-\left|v_{n}\right| e^{-\left|v_{n} \tau\right|}}{\Lambda^{2}-v_{n}^{2}}, \\
\eta(\tau)=\frac{m \gamma \Lambda^{2}}{2} \operatorname{sign}(\tau) e^{-\Lambda|\tau|},
\end{gathered}
$$


and the coefficients can be evaluated as follows:

$$
\begin{aligned}
C_{x}(\Omega) & =-\frac{m \gamma}{2 \pi} \int_{-\infty}^{\infty} d \omega \mathrm{P}\left(\frac{1}{\omega+\Omega} \frac{\omega \Lambda^{2}}{\omega^{2}+\Lambda^{2}}\right) \\
& =-\frac{m \gamma \Lambda^{3}}{2\left(\Omega^{2}+\Lambda^{2}\right)}, \\
C_{p}(\Omega) & =\frac{m \gamma \Omega \Lambda^{2}}{2\left(\Omega^{2}+\Lambda^{2}\right)}, \\
D_{x}(\Omega) & =\frac{m \gamma \Omega \Lambda^{2}}{2\left(\Omega^{2}+\Lambda^{2}\right)} \operatorname{coth}\left(\frac{\hbar \Omega}{2 k_{B} T}\right) .
\end{aligned}
$$

In the first equation above we have used the identity $2 i \int_{0}^{\infty} d \tau \sin (\omega \tau)=\int_{-\infty}^{\infty} d \tau \operatorname{sign}(\tau) e^{i \omega \tau}=2 i \mathrm{P}\left(\frac{1}{\omega}\right)$, where $\mathrm{P}$ denotes the principal value of the integral.

The derivation of the anomalous diffusion coefficient $D_{p}$ is more involved. One has

$$
D_{p}(\Omega)=-\int_{-\infty}^{\infty} \frac{d \omega}{2 \pi} \mathrm{P}\left[\frac{m \gamma \Lambda^{2}}{\omega+\Omega} \frac{\omega}{\omega^{2}+\Lambda^{2}} \operatorname{coth}\left(\frac{\hbar \omega}{2 k_{B} T}\right)\right] .
$$

To perform the principal part integration with the standard trick $\int d \omega \mathrm{P}\left[\frac{f(\omega)}{\omega}\right]=\int d \omega\left[\frac{f(\omega)-f(0)}{\omega}\right]$ we need the numerator to be a polynomial in $\omega$. Inserting the Matsubara representation of the coth in Eq. (30), one finds

$$
\begin{aligned}
& \frac{\pi\left(\Omega^{2}+\Lambda^{2}\right)}{m \gamma \Omega \Lambda^{2}} D_{p}(\Omega) \\
& =-\frac{\pi}{\hbar} \sum_{n=-\infty}^{\infty} \frac{k_{B} T}{\left(\Omega^{2}+v_{n}^{2}\right)} \frac{\left(\Omega^{2}-\Lambda\left|v_{n}\right|\right)}{\Lambda+\left|v_{n}\right|} \\
& =\frac{\pi k_{B} T}{\hbar \Lambda}+\operatorname{Di} \Gamma\left(\frac{\hbar \Lambda}{2 \pi k_{B} T}\right)-\operatorname{Re}\left[\operatorname{Di} \Gamma\left(\frac{i \hbar \Omega}{2 \pi k_{B} T}\right)\right] .
\end{aligned}
$$

The function $\operatorname{Di} \Gamma(z) \equiv \Gamma^{\prime}(z) / \Gamma(z)$ is the logarithmic derivative of the gamma function, and it is plotted in Fig. 1 for both real and imaginary arguments.

The $C_{x}$ term provides a term which strongly renormalizes the harmonic potential frequency. The role of the counterterm $V_{c}$ introduced in the Hamiltonian is exactly to remove this

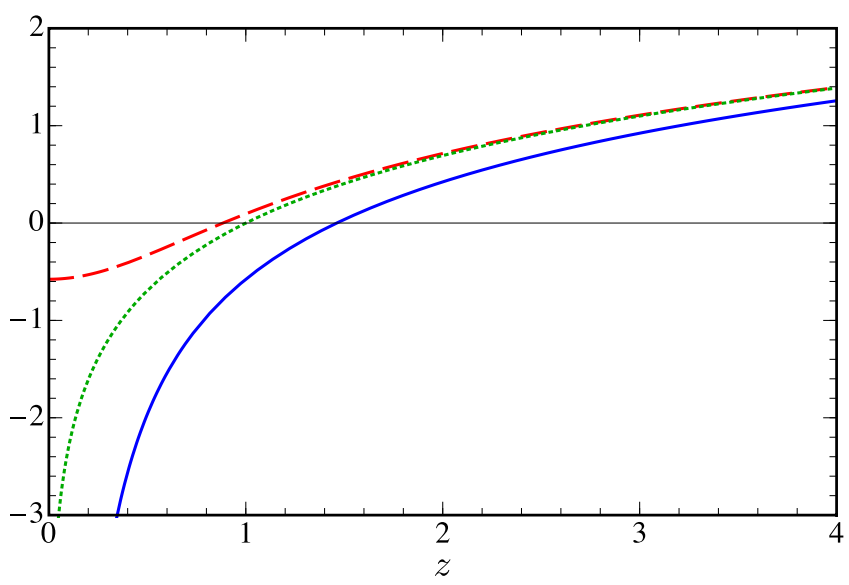

FIG. 1. (Color online) Plots of the adimensional functions $\operatorname{Di} \Gamma(z)$ (continuous line) and $\operatorname{Re}[\operatorname{Di} \Gamma(i z)]$ (dashed line). At large $z$, both functions approach $\ln (z)$ (dotted line).

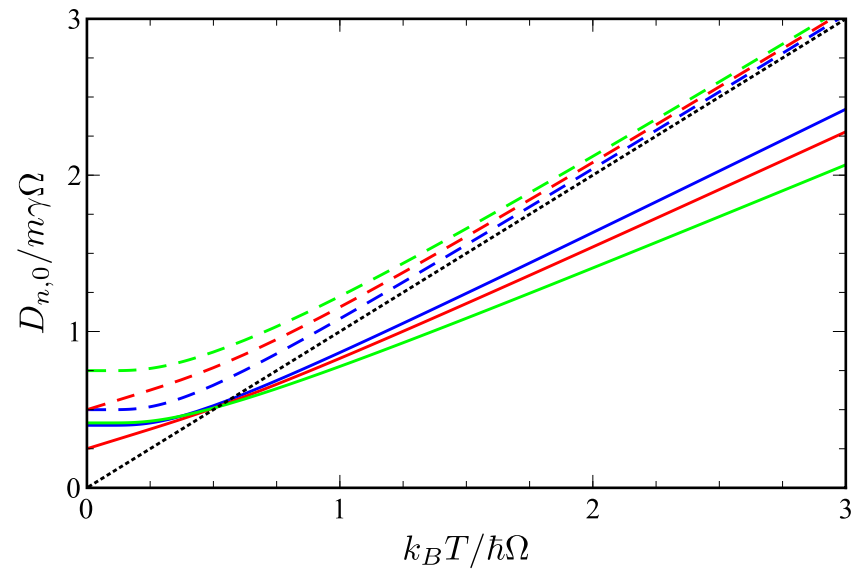

FIG. 2. (Color online) Plot of the coefficients $D_{n, 0}$, which control the decoherence rate of the off-diagonal elements of the density matrix $\rho\left(x_{1}, x_{2}\right)$ in the position basis. The lines represent, respectively, $D_{1,0}=D_{x}$ (blue line), $D_{2,0}=D_{x x}$ (red line), and $D_{3,0}$ (green line). Continuous lines are for $\Lambda=2 \Omega$, dashed lines for $\Lambda=100 \Omega$. In the Caldeira-Leggett limit $k_{B} T / \hbar \gg \Lambda \gg \Omega$, we find $D_{n, 0} \rightarrow$ $m \gamma k_{B} T / \hbar$ (dotted line), independent of $n$.

spurious contribution, and from Eq. (23) we see explicitly that a perfect cancellation is obtained by choosing $V_{c}(x)=$ $-C_{x} x^{2}$. Regarding the other coefficients, as we will see in the following, $C_{p}$ provides momentum damping, $D_{x}$ yields normal momentum diffusion, and $D_{p}$ contributes to anomalous diffusion. The $D_{x}$ term may also be seen as the one responsible for decoherence in the position basis $[3,83,84]$. There, the density matrix may be represented as $\rho\left(x_{1}, x_{2}, t\right)=\left\langle x_{1}|\rho(t)| x_{2}\right\rangle$, and one finds $\partial_{t} \rho\left(x_{1}, x_{2}, t\right)=-D_{x}\left(x_{1}-x_{2}\right)^{2} \rho\left(x_{1}, x_{2}, t\right) / \hbar+\cdots$, so that the off-diagonal components of $\rho$ decohere at a rate directly proportional to the square of the distance between them $\gamma_{x_{1}, x_{2}}^{(1)}=D_{x}\left(x_{1}-x_{2}\right)^{2} / \hbar$ (see Fig. 2).

\section{A. Caldeira-Leggett limit (linear case)}

In the high-temperature and large-cutoff limits $k_{B} T / \hbar \gg$ $\Lambda \gg \Omega$, we may use the series expansions $\operatorname{Di} \Gamma(z)=-z^{-1}-$ $\tilde{\gamma}+\pi^{2} z / 6+O\left(z^{2}\right)$ and $\operatorname{Re}[\operatorname{Di} \Gamma(i z)]=-\tilde{\gamma}+O\left(z^{2}\right)$ (with $\tilde{\gamma}$ the Euler gamma, and real adimensional argument $z$ ) to find

$$
\frac{D_{p}}{\hbar m \Omega}=-\frac{k_{B} T \gamma}{\hbar^{2} \Lambda}+O\left(\frac{\Lambda}{T}\right),
$$

this leading contribution coming from the zero Matsubara frequency term. Apart from a factor $\frac{1}{2}$, due to a different definition of the damping constant $\gamma$, this expression agrees with Eq. (3.409) of Ref. [2], and with Eq. (5.54) of Ref. [3] (mind, however, that the latter one has a minor typo, i.e., this coefficient appears with the wrong sign). Inserting in the ME (23), at high $T$ one finds

$$
\begin{aligned}
\dot{\rho}(t)= & -\frac{i}{\hbar}\left[H_{\mathrm{sys}}, \rho(t)\right]-\frac{i \gamma}{2 \hbar}[x,\{p, \rho(t)\}] \\
& -\frac{m \gamma k_{B} T}{\hbar^{2}}[x,[x, \rho(t)]]+\frac{\gamma k_{B} T}{\hbar^{2} \Lambda}[x,[p, \rho(t)]] .
\end{aligned}
$$

Since $p$ is of order $m \Omega x$ in a harmonic potential, the last term may be neglected as it scales as $\Omega / \Lambda$, and in this way we recover the usual Caldeira-Leggett ME (20). As 
such, in the following we will refer to the regime where $k_{B} T / \hbar \gg \Lambda \gg \Omega$ as the Caldeira-Leggett limit. Note that in the case of a harmonic potential trapping the Brownian particle, or more generally upon neglecting quantum effects for the general nonharmonic potential, the corresponding timedependent equation for the Wigner function in this regime has a particularly simple interpretation (cf. Ref. [1]): it is a FokkerPlank equation for the probability distribution in the phase space of a classical Brownian particle undergoing damped motion with a damping constant $\gamma$ under the influence of a Langevin stochastic noise force $F(t)$. The noise is Gaussian and white, but it fulfills the fluctuation-dissipation relation, i.e., the average of the noise correlation satisfies $\langle F(t+\tau) F(t)\rangle=$ $2 \gamma k_{B} T$. This relation ensures that the stable stationary state of the dynamics is the classical Gibbs-Boltzmann state. In terms of the coefficients entering the master equation, the fluctuationdissipation relation implies that $D_{x} / C_{p}=2 k_{B} T / \hbar \Omega$.

\section{B. Large-cutoff limit (linear case)}

We want to look at the interesting limit $\Lambda \gg \Omega, k_{B} T / \hbar$, with $\Omega \sim k_{B} T / \hbar$; in this case, we find

$$
\begin{aligned}
\dot{\rho}(t)= & -\frac{i}{\hbar}\left[H_{\text {sys }}, \rho(t)\right]-\frac{i \gamma}{2 \hbar}[x,\{p, \rho(t)\}] \\
& -\frac{m \gamma \Omega}{2 \hbar} \operatorname{coth}\left(\frac{\hbar \Omega}{2 k_{B} T}\right)[x,[x, \rho(t)]] \\
& -\frac{D_{p}}{\hbar m \Omega}[x,[p, \rho(t)]] .
\end{aligned}
$$

For large $z$ we have $\operatorname{Di} \Gamma(z) \sim \ln (z)-1 /(2 z)+O\left(z^{-2}\right)$ and $\operatorname{Re}[\operatorname{Di} \Gamma(i z)] \sim \ln (z)+1 /\left(12 z^{2}\right)+O\left(z^{-3}\right), \quad$ and the anomalous diffusion coefficient is proportional to $D_{p} \sim \frac{m \gamma \Omega}{\pi} \ln \left(\frac{\hbar \Lambda}{2 \pi k_{B} T}\right)$. In this limit, we have moreover $D_{x} / C_{p}=\operatorname{coth}\left(\hbar \Omega / 2 k_{B} T\right)$. Equation (33), with the anomalous diffusion coefficient given in Eq. (30), constitutes the main results of this section. As we will argue in Sec. VII and Appendix F, in any practical physical application of the present theory the cutoff energy $\hbar \Lambda$ has a very concrete physical meaning: in a trap the bath frequencies are evidently bound by the trap depth, in an optical lattice by the lowest band's width, and so on.

\section{Ultralow-temperature limit (linear case)}

To conclude the analysis of the linear case, we consider the limit $\Lambda \gg \Omega \gg k_{B} T / \hbar$. Since both Di $\Gamma$ functions in Eq. (30) diverge logarithmically, the temperature drops completely out of the QME, which reads now as

$$
\begin{aligned}
\dot{\rho}(t)= & -\frac{i}{\hbar}\left[H_{\mathrm{sys}}, \rho(t)\right]-\frac{i \gamma}{2 \hbar}[x,\{p, \rho(t)\}] \\
& -\frac{m \gamma \Omega}{2 \hbar}[x,[x, \rho(t)]]-\frac{\gamma}{\hbar \pi} \ln \left(\frac{\Lambda}{\Omega}\right)[x,[p, \rho(t)]] .
\end{aligned}
$$

\section{BM-QME WITH QUADRATIC COUPLING}

Let us now turn to the main subject of this paper: the BornMarkov QME with nonlinear coupling in the particle position. We discuss in detail here the case of quadratic coupling $f(x)=$ $x^{2} / a$, and leave the presentation of the more involved results for a completely general coupling to the Appendix A.

The Heisenberg equation for $x^{2}(\tau)$ yields

$$
\begin{aligned}
x^{2}(-\tau)= & {\left[x \cos (\Omega \tau)-\frac{p}{m \Omega} \sin (\Omega \tau)\right]^{2} } \\
= & x^{2} \cos ^{2}(\Omega \tau)-\frac{\{x, p\}}{m \Omega} \sin (\Omega \tau) \cos (\Omega \tau) \\
& +\frac{p^{2}}{m^{2} \Omega^{2}} \sin ^{2}(\Omega \tau)
\end{aligned}
$$

so that (using the linearity of commutators and anticommutators) one finds

$$
\begin{aligned}
\dot{\rho}(t)= & -\frac{i}{\hbar}\left[H_{S}, \rho(t)\right]-\frac{i C_{x x}}{\hbar a^{2}}\left[x^{2},\left\{x^{2}, \rho(t)\right\}\right]-\frac{i C_{x p}}{\hbar a^{2}}\left[x^{2},\left\{\frac{\{x, p\}}{m \Omega}, \rho(t)\right\}\right]-\frac{i C_{p p}}{\hbar a^{2}}\left[x^{2},\left\{\frac{p^{2}}{m^{2} \Omega^{2}}, \rho(t)\right\}\right] \\
& -\frac{D_{x x}}{\hbar a^{2}}\left[x^{2},\left[x^{2}, \rho(t)\right]\right]-\frac{D_{x p}}{\hbar a^{2}}\left[x^{2},\left[\frac{\{x, p\}}{m \Omega}, \rho(t)\right]\right]-\frac{D_{p p}}{\hbar a^{2}}\left[x^{2},\left[\frac{p^{2}}{m^{2} \Omega^{2}}, \rho(t)\right]\right],
\end{aligned}
$$

with the coefficients $C_{\ldots}$ given by

and the $D_{\ldots}$ by

$$
\begin{aligned}
& C_{x x}=-\int_{0}^{\infty} d \tau \eta(\tau) \cos ^{2}(\Omega \tau), \\
& C_{x p}=\int_{0}^{\infty} d \tau \eta(\tau) \sin (\Omega \tau) \cos (\Omega \tau), \\
& C_{p p}=-\int_{0}^{\infty} d \tau \eta(\tau) \sin ^{2}(\Omega \tau),
\end{aligned}
$$

$$
\begin{aligned}
& D_{x x}=\int_{0}^{\infty} d \tau v(\tau) \cos ^{2}(\Omega \tau), \\
& D_{x p}=-\int_{0}^{\infty} d \tau \nu(\tau) \sin (\Omega \tau) \cos (\Omega \tau), \\
& D_{p p}=\int_{0}^{\infty} d \tau v(\tau) \sin ^{2}(\Omega \tau) .
\end{aligned}
$$


Using $\sin (x) \cos (x)=\sin (2 x) / 2$ and introducing the shorthand notation

$$
c(\Lambda)=\Lambda^{2} /\left(4 \Omega^{2}+\Lambda^{2}\right)
$$

for the cutoff function evaluated at frequency $2 \Omega$, we may exploit the results for $C_{p}$ and $D_{p}$ in the linear case to find

$$
\begin{aligned}
C_{x p}= & \frac{1}{2} \int_{0}^{\infty} d \tau \eta(\tau) \sin (2 \Omega \tau)=\frac{C_{p}(2 \Omega)}{2}=\frac{m \gamma \Omega}{2} c(\Lambda), \\
D_{x p}= & \frac{D_{p}(2 \Omega)}{2}=\frac{m \gamma \Omega}{\pi} c(\Lambda)\left\{\frac{\pi k_{B} T}{\hbar \Lambda}+\operatorname{Di} \Gamma\left(\frac{\hbar \Lambda / 2}{\pi k_{B} T}\right)\right. \\
& \left.-\operatorname{Re}\left[\operatorname{Di} \Gamma\left(\frac{i \hbar \Omega}{\pi k_{B} T}\right)\right]\right\} .
\end{aligned}
$$

Similarly, using $\quad \cos ^{2}(x)=[1+\cos (2 x)] / 2, \quad I_{v} \equiv$ $\int_{0}^{\infty} d \tau \nu(\tau)=m k_{B} T \gamma / \hbar$, and $D_{x}$ for the linear case, one finds

$$
\begin{aligned}
& D_{x x}=\frac{I_{\nu}+D_{x}(2 \Omega)}{2}=\frac{m \gamma \Omega}{2}\left[\frac{k_{B} T}{\hbar \Omega}+c(\Lambda) \operatorname{coth}\left(\frac{\hbar \Omega}{k_{B} T}\right)\right], \\
& D_{p p}=I_{\nu}-D_{x x}=\frac{m \gamma \Omega}{2}\left[\frac{k_{B} T}{\hbar \Omega}-c(\Lambda) \operatorname{coth}\left(\frac{\hbar \Omega}{k_{B} T}\right)\right] .
\end{aligned}
$$

Finally, using $I_{\eta} \equiv \int_{0}^{\infty} d \tau \eta(\tau)=m \gamma \Lambda / 2$, and the derivation for $C_{x}$ in the linear case, we also find

$$
\begin{aligned}
& C_{x x}=-\frac{I_{\eta}}{2}+\frac{C_{x}(2 \Omega)}{2}=-\frac{m \gamma \Lambda\left(2 \Omega^{2}+\Lambda^{2}\right)}{2\left(4 \Omega^{2}+\Lambda^{2}\right)}, \\
& C_{p p}=-I_{\eta}-C_{x x}=-\frac{m \gamma \Omega^{2}}{\Lambda} c(\Lambda) .
\end{aligned}
$$

In analogy with the linear case, the coefficient $C_{x x}$ diverges with the cutoff $\Lambda$, but this poses no problems as $\left[x^{2},\left\{x^{2}, \rho\right\}\right]=$ $\left[x^{4}, \rho\right]$, so this term may always be canceled exactly by an appropriate counterterm $V_{c}(x)=-C_{x x} x^{4} / a^{2}$, representing this time a Lamb shift of the coefficient of the quartic term in the confinement. All other coefficients remain bounded in the limit of $\hbar \Lambda / k_{B} T \rightarrow \infty$, exception made for $D_{x p}$ which exhibits a mild logarithmic divergence, in complete analogy with $D_{p}$ in the linear case. The generalized QME (36), together with the explicit forms of its coefficients, represent a central result of this paper. In the following, we analyze the behavior of the various coefficients in three different limits.

\section{A. Caldeira-Leggett limit (quadratic case)}

In the usual high-temperature limit $k_{B} T / \hbar \gg \Lambda \gg \Omega$, we have

$$
\begin{aligned}
& D_{x x} \approx m \gamma k_{B} T / \hbar, \\
& D_{x p} \approx-m \gamma\left(k_{B} T / \hbar\right)(\Omega / \Lambda) \longrightarrow 0, \\
& D_{p p} \approx-m \gamma \hbar \Omega^{2} /\left(6 k_{B} T\right) \longrightarrow 0,
\end{aligned}
$$

and therefore we obtain

$$
\begin{aligned}
\dot{\rho}(t)= & -\frac{i}{\hbar}\left[H_{\mathrm{sys}}, \rho(t)\right]-\frac{i m \gamma}{2 \hbar}\left[\frac{x^{2}}{a},\left\{\frac{\{x, p\}}{m a}, \rho(t)\right\}\right] \\
& -\frac{m \gamma k_{B} T}{\hbar^{2}}\left[\frac{x^{2}}{a},\left[\frac{x^{2}}{a}, \rho(t)\right]\right],
\end{aligned}
$$

which agrees with the generalized CLME discussed in the Introduction, Eq. (21). In this high-temperature limit, it is easy to identify $C_{x p}$ as being proportional to the momentum damping coefficient, and $D_{x x}$ to the normal momentum diffusion coefficient. In analogy with the linear case, this latter term may also be seen as the one responsible for decoherence in the position basis. The off-diagonal components of $\rho$ are in this way found to decohere at a rate $\gamma_{x_{1}, x_{2}}^{(2)}=D_{x x}\left(x_{1}^{2}-x_{2}^{2}\right)^{2} / \hbar a^{2}$ (see Fig. 2). This is an important result, providing a typical time scale for decoherence of states entangled in position space in presence of a bath coupling of the form $f(x) \propto x^{2}$. In Appendix A, we will provide a general formula which yields the position-space decoherence rate $\gamma_{x_{1}, x_{2}}^{(n)}$ associated to a coupling with an arbitrary power of the system's coordinate $f(x) \propto x^{n}$. Remarkably, and at odds with what is found in Ref. [8], we find that superposition states which are symmetric around the origin (e.g., sharply localized around both $+x_{0}$ and $-x_{0}$ ) will be protected by decoherence in presence of couplings containing only even powers of $n$.

Note also that in this limit we recover again the classical Gibbs-Boltzmann stationary states, and the dynamics satisfies the fluctuation-dissipation relation. Namely, in the case of a harmonic potential, or more generally upon neglecting quantum effects induced by an anharmonic potential, the time-dependent equation for the Wigner function has the interpretation of a Fokker-Plank equation for the probability distribution in the phase space of a classical Brownian particle undergoing damped motion with an $x$-dependent damping $\gamma(x / a)^{2}$ under the influence of a multiplicative Langevin stochastic noise force $F(t)(x(t) / a)$. The noise is Gaussian and white, and it fulfills the fluctuation-dissipation relation, i.e., the average of the noise correlation yields $\langle F(t+$ $\tau) x(t+\tau) F(t) x(t)\rangle=2 \gamma k_{B} T\left\langle x^{2}\right\rangle$. This relation assures that the stable stationary state of the dynamics is the classical Gibbs-Boltzmann state. In terms of the coefficients entering the master equation, the fluctuation-dissipation relation implies that $D_{x x} / C_{x p}=2 k_{B} T / \hbar \Omega$.

\section{B. Large-cutoff limit (quadratic case)}

Taking the more interesting limit $\hbar \Lambda \gg \hbar \Omega, k_{B} T$ simply amounts to setting $c(\Lambda)=1$ in the expression for the various coefficients. In this regime, our QME exhibits several differences in comparison to Eq. (21): (i) the coefficient $C_{p p}$ (a term contributing to a Lamb shift of the trap frequency $\Omega$ ) is suppressed as $\Omega / \Lambda$; (ii) the normal momentum diffusion (or position-basis decoherence) coefficient $D_{x x}$, which is analogous to the $D_{x}$ of the linear case, develops a nontrivial quantum dependence on $\hbar \Omega / k_{B} T$; (iii) the coefficient $D_{x p}$ (which contributes to both the Lamb shift and the anomalous diffusion) becomes log-divergent in $\Lambda$, analogously to $D_{p}$ found in the linear case; (iv) there appears a coefficient $D_{p p}$, which depends on $\hbar \Omega / k_{B} T$, and vanishes for $k_{B} T \gg \hbar \Omega$.

We note here that, in this limit, the coefficients of the QME satisfy the generalized fluctuation-dissipation relations $\left(D_{x x}+D_{p p}\right) / C_{x p}=2 k_{B} T / \hbar \Omega$ and $\left(D_{x x}-D_{p p}\right) / C_{x p}=$ $2 \operatorname{coth}\left(\hbar \Omega / k_{B} T\right)$. Finally, we note that the usual hightemperature limit,in the manner of Caldeira-Leggett, $k_{B} T \gg$ $\hbar \Lambda \gg \hbar \Omega$, should be taken with precaution in the case of nonlinear coupling. Indeed, as we will see in the following (cf. 
Fig. 5), for strong damping the system in a purely harmonic trap may become dynamically unstable at sufficiently large temperatures.

\section{Ultralow-temperature limit (quadratic case)}

The QME equation for $k_{B} T / \hbar \ll \Omega \ll \Lambda$ reads as

$$
\begin{aligned}
\dot{\rho}(t)= & -\frac{i}{\hbar}\left[H_{\mathrm{sys}}, \rho(t)\right]-\frac{i m \gamma}{2 \hbar}\left[\frac{x^{2}}{a},\left\{\frac{\{x, p\}}{m a}, \rho(t)\right\}\right] \\
& -\frac{m \gamma \Omega}{2 \hbar}\left[\frac{x^{2}}{a},\left[\frac{x^{2}}{a}-\frac{p^{2}}{m^{2} \Omega^{2} a}, \rho(t)\right]\right] \\
& -\frac{m \gamma}{\hbar \pi} \ln \left(\frac{\Lambda}{2 \Omega}\right)\left[\frac{x^{2}}{a},\left[\frac{\{x, p\}}{m a}, \rho(t)\right]\right] .
\end{aligned}
$$

As expected, the temperature drops out of the equation, and the $D_{x p}$ term is log-divergent in the cutoff $\Lambda$.

\section{WIGNER FUNCTION APPROACH AND STATIONARY SOLUTIONS}

The quantum master equation for the density matrix $\rho$ can be particularly well analyzed in terms of the Wigner function $W$. To this aim, it is useful to introduce the operators $x_{ \pm}=$ $x \pm \frac{i \hbar}{2} \frac{\partial}{\partial p}$ and $p_{ \pm}=p \pm \frac{i \hbar}{2} \frac{\partial}{\partial x}$, which satisfy the commutation rules

$$
\begin{aligned}
& {\left[x_{+}, x_{-}\right]=\left[p_{+}, p_{-}\right]=0,} \\
& {\left[x_{+}, p_{-}\right]=-\left[x_{-}, p_{+}\right]=i \hbar .}
\end{aligned}
$$

The formal substitutions [see Eqs. (4.5.11) of [1]] are of great use in the following:

$$
\begin{aligned}
& \hat{x} \rho \rightarrow x_{+} W, \quad \hat{p} \rho \rightarrow p_{-} W, \\
& \rho \hat{x} \rightarrow x_{-} W, \quad \rho \hat{p} \rightarrow p_{+} W .
\end{aligned}
$$

We note here that, while in the previous sections $x$ and $p$ stood for the usual noncommuting operators, from now on the same symbols will be used to represent the commuting variables of the Wigner function $W(x, p)$.

\section{A. Linear case}

Let us first analyze the case of linear coupling. When $f(x)=x$, the QME for general $\Omega, \Lambda$, and $T$ in terms of the Wigner function reads as ${ }^{2}$

$\dot{W}=\left[m \Omega^{2} \partial_{p} x-\frac{\partial_{x} p}{m}+\frac{2 C_{p}}{m \Omega} \partial_{p} p+\hbar D_{x} \partial_{p}^{2}-\frac{\hbar D_{p}}{m \Omega} \partial_{x} \partial_{p}\right] W$.

The stationary solution to this equation may be found by inserting a generic quadratic ansatz

$$
W_{\text {st }} \propto \exp \left[-\left(\sigma_{p} \frac{p^{2}}{2 m}+\sigma_{x} \frac{m \Omega^{2} x^{2}}{2}\right) /\left(k_{B} \tilde{T}\right)\right]
$$

with real parameters $\sigma_{p}$ and $\sigma_{x}$, and equating independently the coefficients of $x^{2}$ and $p^{2}$ to zero in the resulting equation.

\footnotetext{
${ }^{2}$ Note that $[\hat{p}, \rho] \hat{x}=\left[\left(p_{-}-p_{+}\right) \rho\right] \hat{x}=x_{-}\left(p_{-}-p_{+}\right) W$.
}

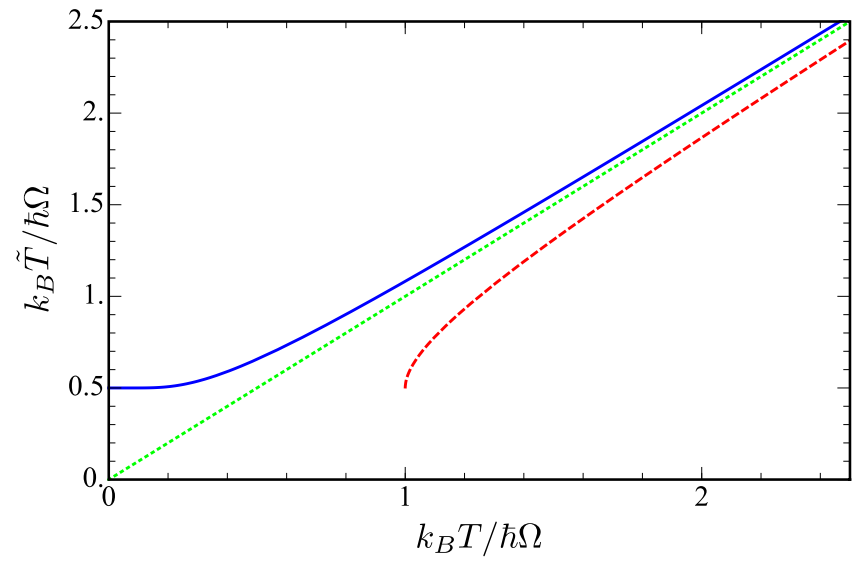

FIG. 3. (Color online) Effective temperatures as obtained through the complete quantum treatment [Eq. (45)] (blue line), and by means of an oversimplified approximation discussed in Appendix E [Eq. (E5)] (red line). The green line is the high- $T$ result $\tilde{T}=T$.

In the oversimplified high- $T$ limit $k_{B} T \gg \hbar \Lambda \gg \hbar \Omega$, in the manner of Caldeira-Leggett, one would set $D_{x}=m \gamma k_{B} T / \hbar$ and $D_{p}=0$, and find in this way $\sigma_{p}=\sigma_{x}=1$, and $\tilde{T}=T$. By retaining instead the complete expression of all terms in the equation (and, in particular, a nonzero $D_{p}$ ), we find that the stationary Wigner function is obtained by choosing $\sigma_{p}=1$ and

$$
\sigma_{x}=\frac{1}{1-2 D_{p} /\left(m \Omega^{2} \operatorname{coth}\left[\hbar \Omega / 2 k_{B} T\right]\right)},
$$

yielding an effective temperature

$$
\tilde{T}=\frac{\hbar \Omega}{2 k_{B}} \operatorname{coth}\left(\frac{\hbar \Omega}{2 k_{B} T}\right) .
$$

This result is shown in Fig. 3. A number of interesting conclusions may now be drawn.

First of all, a careful treatment of the equation at low $T$ yields an effective temperature which saturates to the zero-point motion energy. When $\sigma_{p}=\sigma_{x}=1$, the Gaussian stationary solution with an effective temperature $\tilde{T}$ as given by the quantum result (45) corresponds to the exact quantum thermal Gibbs-Boltzmann density matrix of a harmonic oscillator (the system) at the temperature $T$. In this case, the contours of the stationary distributions are circles of radius $\sqrt{2 k_{B} \tilde{T} / \hbar \Omega}$ for arbitrary $T$ (i.e., of radius 1 at $T=0$ ).

More generally, in units of the normalized standard deviations

$$
\begin{aligned}
& \delta_{x}=2 \sqrt{\frac{m \Omega^{2}\left\langle x^{2}\right\rangle_{\mathrm{st}}}{2 \hbar \Omega}}=\sqrt{\frac{2 k_{B} \tilde{T}}{\hbar \Omega \sigma_{x}}}, \\
& \delta_{p}=2 \sqrt{\frac{\left\langle p^{2}\right\rangle_{\mathrm{st}}}{2 m \hbar \Omega}}=\sqrt{\frac{2 k_{B} \tilde{T}}{\hbar \Omega \sigma_{p}}},
\end{aligned}
$$

the Heisenberg uncertainty relation requires that

$$
\delta_{x} \delta_{p} \geqslant 1
$$

i.e., that the contour of the distribution encircles an area not smaller than $\pi$. An important effect of $D_{p}$ is that it allows for a contraction of the distribution in $x$ versus $p$. 


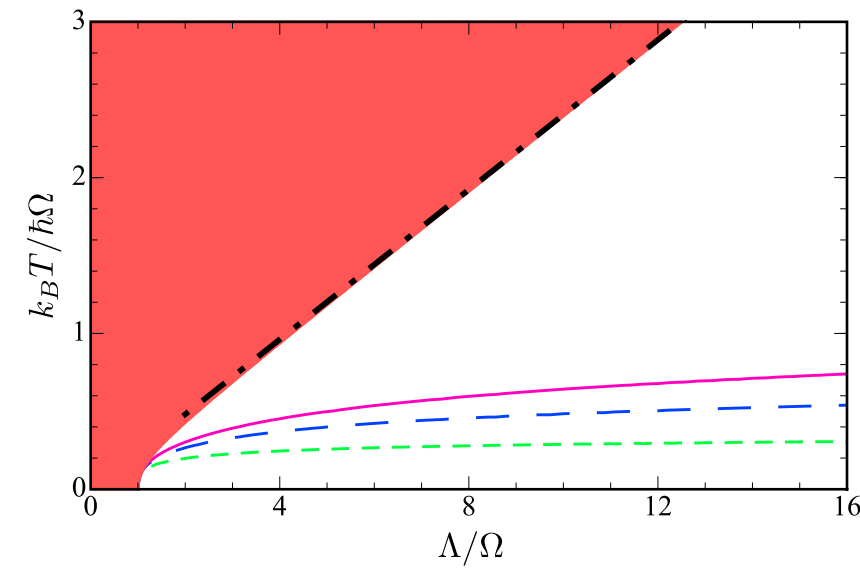

FIG. 4. (Color online) Minimal temperature for the fulfillment of the Heisenberg uncertainty principle for an Ohmic spectral function with LD cutoff in the linear case, for $\gamma / \Omega=0.1,0.5,1$ (from bottom to top). In the red region, the gas displays effective "heating" and a quenched aspect ratio in $p$ relative to $x$ (i.e., $\delta_{x} / \delta_{p}>1$ ). The black, dotted-dashed line is the asymptotic approximation to the boundary of unit aspect ratio $T=\alpha_{(1)} \Lambda$.

The Heisenberg uncertainty principle then puts an important constraint on our theory, forcing us to exclude the region where the inequality is violated. In Fig. 4, we illustrate this region of validity, as obtained by inserting Eq. (44) in Eq. (47): for any $\Lambda>\Omega$, we find that there exists a critical temperature below which the Heisenberg uncertainty principle is violated. Similar squeezing effects have been discussed [82] in the literature in the context of the so-called Ullersma model [81]. At $T=0$, the Heisenberg principle requires $\Lambda<\Omega$.

Interestingly, in the linear case there are no log-corrections to $\tilde{T}$ coming from the log-divergent term $D_{p} . D_{p}$ grows with the cutoff, and at very large values $\sigma_{x}$ diverges (i.e., $\delta_{x}^{2}$ approaches zero) and becomes negative, yielding a nonnormalizable solution. However, this bound always lies beyond the one set by the Heisenberg principle, which requires $\delta_{x} \delta_{p} \geqslant 1$.

We may say that the quantum particle immersed in the bath experiences an effective "heating" if the phase-space area encircled by the normalized standard deviations is larger than the one a quantum Gibbs-Boltzmann (GB) distribution would occupy at the same temperature. Since $\left\langle E_{k}\right\rangle_{\mathrm{GB}}\left\langle E_{p}\right\rangle_{\mathrm{GB}}=$ $\left(k_{B} \tilde{T} / 2\right)^{2}$, the system is effectively heated if

$$
\delta_{x} \delta_{p}>\operatorname{coth}\left(\frac{\hbar \Omega}{2 k_{B} T}\right)
$$

or, equivalently, $\sigma_{x} \sigma_{p}<1$. Since $\sigma_{p}=1$ in the linear case, this amounts to requiring $D_{p}<0$, which remarkably does not depend on $\gamma$. Asymptotically, we have $T>\alpha_{(1)} \Lambda+O(\Omega / T)$, with $\alpha_{(1)} \approx 0.24$ solution of the implicit equation

$$
\pi \alpha_{(1)}+\operatorname{Di} \Gamma\left(1 / 2 \pi \alpha_{(1)}\right)+\tilde{\gamma}=0 .
$$

Finally, we consider the aspect ratio of the phase-space contour described by the standard deviations. Since $\sigma_{p}$ always equals unity in the linear case, it is easy to see that we have a quenched aspect ratio in $x$, relative to $p$ (i.e., $\delta_{x} / \delta_{p}<1$ ) in the "cooling" region, and the opposite situation $\left(\delta_{x} / \delta_{p}>1\right)$ in the "heating" region. In fact, the line separating "heating" region from the "cooling" region corresponds to the regime where $D_{p}=0$. In this case, the Wigner function is exactly given by a Gaussian with effective temperature $\tilde{T}$, and circular shape of the distribution $\left(\delta_{p}=\delta_{x}\right)$; it corresponds precisely to the quantum thermal Gibbs-Boltzmann density matrix.

It should be noted that, when deriving the stationary solutions from a perturbative treatment of the master equation to order $2 n$ in the bath-system coupling constant $\kappa_{k}$, one gets a reduced equilibrium state which is exact to order $2 n-2$, and contains some (but not all) terms of the order- $2 n$ solutions. The overall error is therefore of order $\left(\kappa_{k}\right)^{2 n}$ itself, as pointed out by Fleming and Cummings [85] (for discussion of the nature of exact reduced equilibrium states, see also [86]). Indeed, the violation of the Heisenberg uncertainty principle we observe within our BM-QME, which is of second order in $\kappa_{k}$, is driven by the unphysical logarithmic divergence of $D_{p}$, which is itself proportional to $\gamma$, i.e., to $\kappa_{k}^{2}$. Obviously, if the exact master equation is used, then Heisenberg uncertainty violation cannot occur in any parameter regime, ergo this violation is not physical, but is rather a result of applied approximations. On the other hand, it is to be expected that both the degree of cooling and squeezing in the considered quantum stochastic process should be bounded from below, and the Heisenberg uncertainty violation gives a reasonable estimate of this bound.

\section{B. Quadratic case}

We turn now to the most interesting case, the quadratic case with $f(x)=x^{2} / a$. We consider the complete equation, obtained using the results in Sec. IV B, and as usual we reabsorb the (linearly divergent in $\Lambda$ ) contribution coming from the $C_{x x}$ term in the Hamiltonian $H_{\text {sys }}$, by requiring $V_{c}(x)=-C_{x x} f(x)^{2}$. The equation of motion for the Wigner function of a harmonically confined particle reads then as

$$
\begin{aligned}
\dot{W}= & -\frac{i}{\hbar}\left[\frac{p_{-}^{2}-p_{+}^{2}}{2 m}+V\left(x_{+}\right)-V\left(x_{-}\right)\right] W-\left(x_{+}^{2}-x_{-}^{2}\right)\left[\frac{i C_{x p}\left(\left\{x_{+}, p_{-}\right\}+\left\{x_{-}, p_{+}\right\}\right)}{\hbar m \Omega a^{2}}+\frac{i C_{p p}\left(p_{-}^{2}+p_{+}^{2}\right)}{\hbar m^{2} \Omega^{2} a^{2}}\right. \\
& \left.+\frac{D_{x x}\left(x_{+}^{2}-x_{-}^{2}\right)}{\hbar a^{2}}+\frac{D_{x p}\left(\left\{x_{+}, p_{-}\right\}-\left\{x_{-}, p_{+}\right\}\right)}{\hbar m \Omega a^{2}}+\frac{D_{p p}\left(p_{-}^{2}-p_{+}^{2}\right)}{m^{2} \Omega^{2} \hbar a^{2}}\right] W \\
= & \left\{-\frac{\partial_{x} p}{m}+m \Omega^{2} \partial_{p} x+\frac{8 C_{x p}}{m \Omega a^{2}}\left[\partial_{p} p x^{2}+\frac{\hbar^{2}}{4} \partial_{p}^{2}\left(\partial_{x} x-1\right)\right]+\frac{C_{p p}}{(m \Omega a)^{2}}\left(4 \partial_{p} x p^{2}-\hbar^{2} \partial_{p} \partial_{x}^{2} x+2 \hbar^{2} \partial_{p} \partial_{x}\right)\right. \\
& \left.+\frac{4 \hbar D_{x x} \partial_{p}^{2} x^{2}}{a^{2}}+\frac{4 \hbar D_{x p}\left(\partial_{p}^{2} x p-\partial_{p} \partial_{x} x^{2}+\partial_{p} x\right)}{m \Omega a^{2}}-\frac{4 \hbar D_{p p}\left(\partial_{x} x-1\right) \partial_{p} p}{m^{2} \Omega^{2} a^{2}}\right\} W .
\end{aligned}
$$


Interestingly, the Gaussian ansatz (43) would provide a stationary solution to the above equation if we neglected the terms proportional to $C_{p p}$ and $D_{x p}$. Remembering that $D_{x x}-$ $D_{p p}=2 C_{x p} \operatorname{coth}\left(\hbar \Omega / k_{B} T\right)$, the stationary solution is found when $\sigma_{p}=\sigma_{x}=1$ and

$$
k_{B} \tilde{T} \stackrel{\left(C_{p p}=D_{x p}=0\right)}{=} \frac{\hbar \Omega}{2} \operatorname{coth}\left(\frac{\hbar \Omega}{2 k_{B} T}\right),
$$

which coincides with the result found above for the linear case [Eq. (45)]. Unfortunately, however, $D_{x p}$ is generally not negligible, as for example it diverges logarithmically with the cutoff $\Lambda$. In order to incorporate the neglected terms, one may try to generalize the ansatz by including in the exponent terms proportional to higher polynomials in $x^{2}$ and $p^{2}$ (i.e., terms such as $x^{4}, x^{2} p^{2}$, or $p^{4}$ ), but no closed solution can be be found in this way, as moments of a given order always couple with higher ones.

The contributions higher than quadratic can, however, be reasonably taken into account by means of the so-called selfconsistent Gaussian (or pairing) approximation [87,88]. The $D_{x p}$ term is proportional to

$$
\begin{aligned}
\partial_{p}^{2} x p-\partial_{p} \partial_{x} x^{2}+\partial_{p} x & \simeq \partial_{p}^{2}\langle x p\rangle_{\mathrm{st}}-\partial_{p} \partial_{x}\left\langle x^{2}\right\rangle_{\mathrm{st}}+\partial_{p} x \\
& =-\partial_{p} \partial_{x} \frac{k_{B} \tilde{T}}{\sigma_{x} m \Omega^{2}}+\partial_{p} x .
\end{aligned}
$$

As a general rule, averages of odd functions or partial derivatives vanish when performed with respect to the Gaussian distribution (43). Similarly, the $C_{p p}$ term contributes

$$
4 \partial_{p} x p^{2}-\hbar^{2} \partial_{p} \partial_{x}^{2} x+2 \hbar^{2} \partial_{p} \partial_{x} \approx \frac{4 m k_{B} \tilde{T}}{\sigma_{p}} \partial_{p} x+2 \hbar^{2} \partial_{p} \partial_{x}
$$

as (mixed) derivatives of order higher than two vanish in this approximation. In this way, we get the two equations

$$
\begin{gathered}
\delta_{p}^{2}=\frac{\delta_{x}^{2}}{\zeta}+\Gamma c_{p p}\left(\frac{\delta_{x}^{2} \delta_{p}^{2}}{2}-1\right), \\
\delta_{x}^{2} \delta_{p}^{2}=\frac{\delta_{x}^{2} d_{x x}-\delta_{p}^{2} d_{p p}}{c_{x p}}-1 .
\end{gathered}
$$

To simplify notation, we have introduced the normalized damping $\Gamma \equiv 2 \hbar \gamma /\left(m \Omega^{2} a^{2}\right)$, the adimensional variables $c_{x p}=2 C_{x p} /(m \gamma \Omega)$ (and similarly for $\left.c_{p p}, d_{x p}, \ldots\right)$, and the quantity $\zeta=1 /\left(1+2 \Gamma d_{x p}\right)$.

The two coupled equations (54) and (55) may be combined to obtain a single quadratic equation determining, e.g., $\delta_{x}^{2}$, from which we may then extract $\delta_{p}^{2}$. The quadratic equation has two solutions, and the correct one may be selected by looking at its behavior in the regime $\Omega \ll k_{B} T / \hbar \ll \Lambda$. The $(-)$ solution unphysically tends towards zero there. On the other hand, the (+) solution correctly yields $\delta_{x}^{2} \sim 2 k_{B} T / \hbar \Omega$, i.e., an effective temperature $\tilde{T} \sim T$. At odds with the linear case, however, $\tilde{T}$ strongly deviates from $T$ when $T \sim O(\Lambda / \Omega)$.

A detailed phase diagram for the present case of quadratic coupling is presented in Fig. 5. The Heisenberg principle requires $\delta_{x} \delta_{p} \geqslant 1$, a condition which gives rise to a minimal acceptable temperature which grows as $T_{\min } \sim \ln (\Lambda)$ for large $\Lambda / \Omega$, in close analogy to the linear case. The Heisenberg bound is shown in Fig. 5(a), together with the region where the gas experiences an effective heating, or cooling, with respect to its Gibbs-Boltzmann counterpart.

The corresponding degree of deformation of the phasespace distribution, as measured by the logarithm of the aspect ratio $\ln \left(\delta_{x}^{2} / \delta_{p}^{2}\right)=\ln \left(\sigma_{p} / \sigma_{x}\right)$, is shown in Fig. 5(b). At small temperatures, we observe the emergence of a region (below the magenta, dotted-dashed lines) where $\delta_{x}^{2}<1$, i.e., of genuine quantum squeezing. Notice that, for damping $\Gamma \gtrsim 0.1$, at large temperatures the aspect ratio of the distribution displays a very sharp increase; beyond a certain point, the solution of Eqs. (54) and (55) yields a value for the fluctuations $\delta_{x}^{2}$ which diverges and turns negative, a clearly unphysical feature signaling the breakdown of the Gaussian ansatz in that region.

It may be noticed by comparing Figs. 5(a) and 5(b) that, as in the linear case, the Gibbs-Boltzmann boundary coincides with the one of unit aspect ratio, a condition which again is independent of $\Gamma$. This may be explicitly checked by employing the trial GB solution $\delta_{x}^{2}=\delta_{p}^{2}=\operatorname{coth}\left(\hbar \Omega / 2 k_{B} T\right)$, which is an identical solution of Eq. (55) for every $\{\Lambda, \Omega, T\}$, and a solution of Eq. (54) for every $\Gamma$ provided that $T=$ $\alpha_{(2)} \Lambda+O(\Omega / T)$, with $\alpha_{(2)} \approx 0.189$ satisfying the implicit equation

$$
\pi \alpha_{(2)}+2\left[\operatorname{Di} \Gamma\left(1 / 2 \pi \alpha_{(2)}\right)+\tilde{\gamma}\right]=0 .
$$

At odds with the linear case seen above, the equations for a quadratic coupling determine the two ratios $\delta_{x}^{2} \propto \tilde{T} / \sigma_{x}$ and $\delta_{p}^{2} \propto \tilde{T} / \sigma_{p}$, but do not provide an explicit expression for $\tilde{T}$, $\sigma_{x}$, and $\sigma_{p}$ separately, leaving therefore open various possible applications of this theory.

As an example, we may fix $\tilde{T}$ in accordance to the standard formula for the quantum mechanical harmonic oscillator [Eq. (45)] and then interpret $\sigma_{p}$ and $\sigma_{x}$ as quantum corrections to the inverse mass $1 / m$ and the spring constant $m \Omega^{2}$. Such "renormalization" should be used if we considered the starting model as a fundamental quantum field theoretic construct.

Alternatively, one may set, say, $\sigma_{p}=1$, and consider quantum modification of the effective temperature, and the spring constant. From Eq. (54), one finds in this way

$$
k_{B} \tilde{T}=\frac{\hbar \Omega}{2} \frac{\delta_{x}^{2} / \zeta-\Gamma c_{p p}}{1-\Gamma c_{p p} \delta_{x}^{2} / 2} .
$$

Similarly as in the case of the linear coupling, one needs to examine the nature of Heisenberg uncertainty pathologies in the present quadratic case. Obviously, the exact stationary state should not violate the Heisenberg uncertainty inequality. In the quadratic case, however, the exact solution is not known, and the results of Ref. [85] cannot be applied directly. The pathologies may result from solutions being of mixed order as in Ref. [85], or from the non-Gaussian form of the unknown exact solution. In any case, the pathologies signal the invalidity of applied approximations and offer a reasonable bound for the degree of cooling and squeezing in the considered quantum stochastic process. 
$\Gamma=0.1$

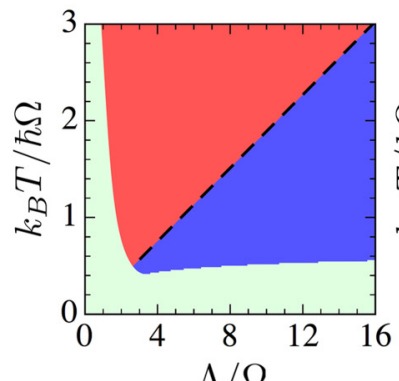

$\Lambda / \Omega$

$\Gamma=0.1$

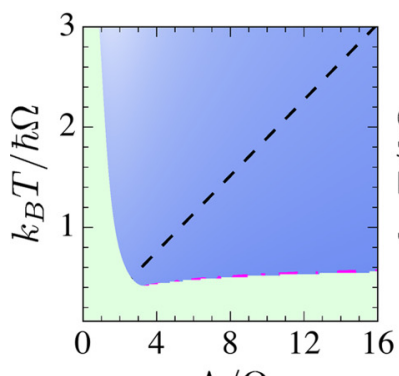

$\Lambda / \Omega$

$\Gamma=0.1$

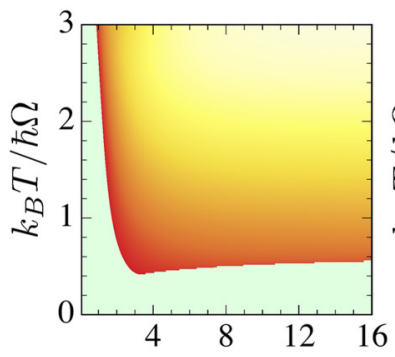

$\Lambda / \Omega$
$\Gamma=0.5$

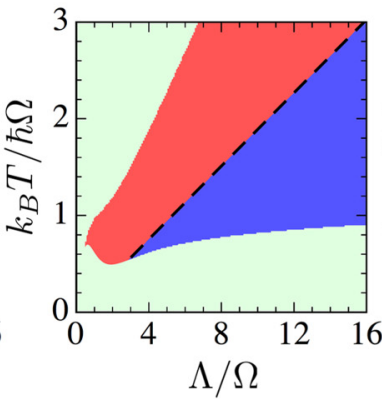

$\Gamma=0.5$

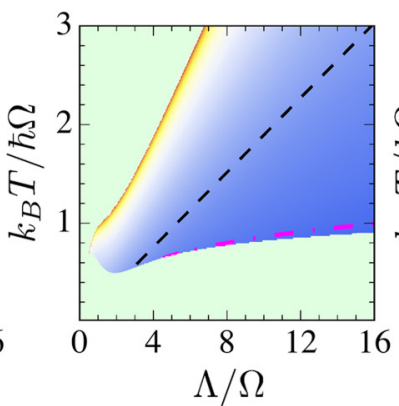

$\Gamma=0.5$

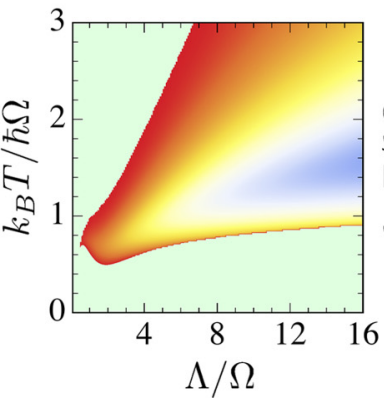

$\Gamma=1$
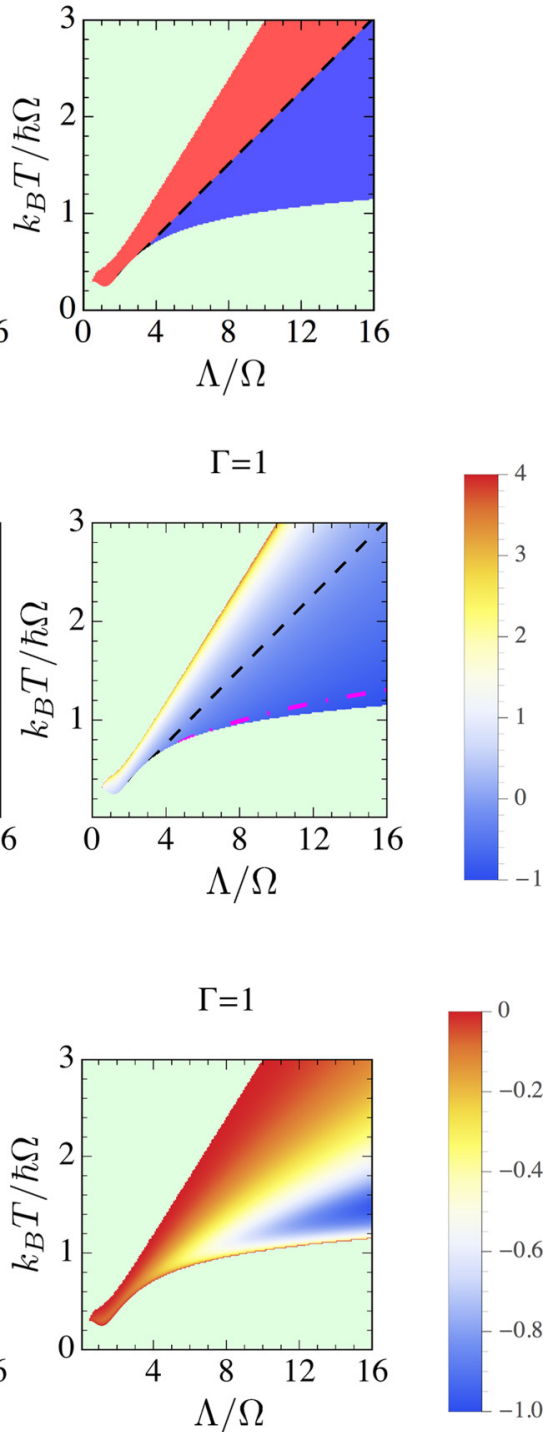

FIG. 5. (Color online) Phase diagram of our equation for a quadratic coupling, under the self-consistent Gaussian approximation. From left to right, plots are for $\Gamma=0.1,0.5,1$. Top (a): the gas experiences an effective "cooling" in the blue regions, and an effective "heating" in the red regions. Center (b): density plot of the logarithm of the aspect ratio $\ln \left(\delta_{x}^{2} / \delta_{p}^{2}\right)$. Bottom (c): maximum of the real part of the eigenvalues of the matrix of coefficients of the linear system defined in Eq. (65). In the green regions, one of the validity conditions is violated, i.e., either the Heisenberg principle is not satisfied, or one of the eigenvalues of the stability equations becomes positive, or fluctuations $\delta_{x}^{2}$ and $\delta_{p}^{2}$ are complex numbers. The black dashed lines are the boundaries of unity aspect ratio, where $\delta_{x}^{2}=\delta_{p}^{2}$. In this way, we see we have "cooling" for $\delta_{x}^{2} / \delta_{p}^{2}<1$, and "heating" for $\delta_{x}^{2} / \delta_{p}^{2}>1$. We have quantum squeezing with $\delta_{x}^{2}<1$ below the magenta dotted-dashed lines, while $\delta_{p}^{2}$ is never smaller than 1 in the allowed region.

\section{NEAR-EQUILIBRIUM DYNAMICS IN SELF-CONSISTENT GAUSSIAN APPROXIMATION}

In this last section before Conclusions, we investigate the near-equilibrium dynamics and stability of stationary solutions found in the previous section. We use the self-consistent Gaussian approximation, which actually is exact in the case of linear coupling provided the initial state was Gaussian.

\section{A. Linear case}

It is elementary to derive the equations for the first and second moments of the Wigner distribution: these moments characterize the Gaussian state fully, and in the linear case form two closed systems of linear equations:

$$
\begin{aligned}
& \langle\dot{x}\rangle=\langle p\rangle / m, \\
& \langle\dot{p}\rangle=-m \Omega^{2}\langle x\rangle-\frac{2 C_{p}}{m \Omega}\langle p\rangle,
\end{aligned}
$$

and

$$
\begin{aligned}
& \left\langle\dot{\left.x^{2}\right\rangle}=2\langle x p\rangle / m,\right. \\
& \langle\dot{x p}\rangle=\frac{\left\langle p^{2}\right\rangle}{m}-m \Omega^{2}\left\langle x^{2}\right\rangle-\frac{2 C_{p}}{m \Omega}\langle x p\rangle-\frac{\hbar D_{p}}{m \Omega}, \\
& \left\langle\dot{\left.p^{2}\right\rangle}=-2 m \Omega^{2}\langle x p\rangle-\frac{4 C_{p}}{m \Omega}\left\langle p^{2}\right\rangle+2 \hbar D_{x} .\right.
\end{aligned}
$$


Clearly, the solutions tend to their stable stationary values $\langle x\rangle_{\mathrm{st}}=\langle p\rangle_{\mathrm{st}}=\langle x p\rangle_{\mathrm{st}}=0,\left\langle p^{2}\right\rangle_{\mathrm{st}}=\hbar m \Omega D_{x} / 2 C_{p}$, and $\left(m^{2} \Omega^{2}\right)\left\langle x^{2}\right\rangle_{\text {st }}=\hbar\left(m \Omega D_{x} / 2 C_{p}-D_{p} / \Omega\right)$. The only constraint is imposed by the Heisenberg principle

$$
\frac{m \Omega^{2}\left\langle x^{2}\right\rangle}{2} \frac{\left\langle p^{2}\right\rangle}{2 m} \geqslant\left(\frac{\hbar \Omega}{4}\right)^{2} .
$$

The equations for $\left\langle x^{2}\right\rangle_{\mathrm{st}}$ and $\left\langle p^{2}\right\rangle_{\mathrm{st}}$ and the resulting Heisenberg bound coincides with the one found for $\sigma_{x}, \sigma_{p}$, and $\delta_{x} \delta_{p}$ in Sec. V A, a fact which should not surprise, as we have seen that a Gaussian ansatz was providing an exact solution of the problem.

\section{B. Quadratic case}

In this case, the Gaussian ansatz provides only an approximate solution. Again, the first and second moments of the Wigner distribution characterize the Gaussian state fully, but this time they couple to higher moments, so that Wick (Gaussian) decorrelation techniques have to be used. We obtain for the first moments

$$
\begin{aligned}
\langle\dot{x}\rangle= & \langle p\rangle / m, \\
\langle\dot{p}\rangle= & -m \Omega^{2}\langle x\rangle-\frac{8 C_{x p}}{m \Omega a^{2}}\left\langle x^{2} p\right\rangle-\frac{4 C_{p p}}{(m \Omega a)^{2}}\left\langle x p^{2}\right\rangle \\
& -\frac{4 \hbar D_{x p}}{m \Omega a^{2}}\langle x\rangle-\frac{4 \hbar D_{p p}}{m^{2} \Omega^{2} a^{2}}\langle p\rangle .
\end{aligned}
$$

The Wick's theorem allows us to replace $\left\langle x^{2} p\right\rangle=\langle x\rangle^{2}\langle p\rangle+$ $2\left\langle\Delta_{x} \Delta_{p}\right\rangle\langle x\rangle+\left\langle\Delta_{x}^{2}\right\rangle\langle p\rangle$, and similarly for $\left\langle x p^{2}\right\rangle$, where we represent the Gaussian random variables $x=\langle x\rangle+\Delta_{x}, p=$ $\langle p\rangle+\Delta_{p}$. We obtain thus

$$
\begin{aligned}
\langle\dot{p}\rangle= & -m \Omega^{2}\langle x\rangle-\frac{8 C_{x p}\left(\langle x\rangle^{2}+\left\langle\Delta_{x}^{2}\right\rangle\right)}{m \Omega a^{2}}\langle p\rangle \\
& -\frac{4 C_{p p}\left(\langle p\rangle^{2}+\left\langle\Delta_{p}^{2}\right\rangle\right)}{m^{2} \Omega^{2} a^{2}}\langle x\rangle-\frac{4 \hbar D_{x p}}{m \Omega a^{2}}\langle x\rangle \\
& -\frac{4 \hbar D_{p p}}{m^{2} \Omega^{2} a^{2}}\langle p\rangle-\frac{8\left\langle\Delta_{x} \Delta_{p}\right\rangle}{m^{2} \Omega^{2} a^{2}}\left(C_{p p}\langle p\rangle+2 m \Omega C_{x p}\langle x\rangle\right) .
\end{aligned}
$$

These equations have a stable stationary solution $\langle x\rangle_{\mathrm{st}}=$ $\langle p\rangle_{\text {st }}=0$, provided that they describe a damped harmonic oscillator. If such a solution exists, in its vicinity we may identify $\left\langle\Delta_{x}^{2}\right\rangle_{\mathrm{st}}=\left\langle x^{2}\right\rangle_{\mathrm{st}}=\delta_{x}^{2} \hbar /(2 m \Omega)$ and $\left\langle\Delta_{p}^{2}\right\rangle_{\mathrm{st}}=\left\langle p^{2}\right\rangle_{\mathrm{st}}=$ $\hbar m \Omega \delta_{p}^{2} / 2$ (since by hypothesis the first moments are zero), and we may neglect the quadratic terms $\langle x\rangle^{2}$ and $\langle p\rangle^{2}$ and the crossed fluctuation term $\left\langle\Delta_{x} \Delta_{p}\right\rangle$, to obtain the two simultaneous conditions

$$
1+\Gamma d_{x p}+\Gamma c_{p p} \delta_{p}^{2} / 2 \geqslant 0, \quad c_{x p} \delta_{x}^{2}+d_{p p} \geqslant 0 .
$$

These, in turn, depend self-consistently on the equations for the second moments

$$
\begin{aligned}
\left\langle\dot{x^{2}}\right\rangle= & \frac{2}{m}\langle x p\rangle \\
\langle\dot{x p}\rangle= & \frac{\left\langle p^{2}\right\rangle}{m}-m \Omega^{2}\left\langle x^{2}\right\rangle-\frac{8}{m \Omega a^{2}}\left[C_{x p}\left\langle x^{3} p\right\rangle+\hbar D_{x p}\left\langle x^{2}\right\rangle\right] \\
& -\frac{1}{m^{2} \Omega^{2} a^{2}}\left[C_{p p}\left(4\left\langle x^{2} p^{2}\right\rangle-2 \hbar^{2}\right)+8 \hbar D_{p p}\langle x p\rangle\right],
\end{aligned}
$$

$$
\begin{aligned}
\left\langle\dot{p}^{2}\right\rangle= & -2 m \Omega^{2}\langle x p\rangle-\frac{4 C_{x p}}{m \Omega a^{2}}\left(4\left\langle x^{2} p^{2}\right\rangle+\hbar^{2}\right) \\
& -\frac{8 C_{p p}}{m \Omega a^{2}}\left\langle x p^{3}\right\rangle+\frac{8 \hbar D_{x x}}{a^{2}}\left\langle x^{2}\right\rangle-\frac{8 \hbar D_{p p}}{m^{2} \Omega^{2} a^{2}}\left\langle p^{2}\right\rangle .
\end{aligned}
$$

From the first equation, we see that if a stable stationary solution exists then $\langle x p\rangle_{\mathrm{st}}=0$. The quartic terms may be decomposed as above, using the Wick's method, and in this way one may compute the stationary solution. A straightforward calculation then shows that in the stationary state $\left\langle x^{2}\right\rangle_{\text {st }}$ and $\left\langle p^{2}\right\rangle_{\text {st }}$ satisfy the same two equations found in the preceding section [Eqs. (54) and (55)]. To check the stability of the steady state, we write $\left\langle x^{2}\right\rangle=\left\langle x^{2}\right\rangle_{\mathrm{st}}+\Delta_{x^{2}}$, $\left\langle p^{2}\right\rangle=\left\langle p^{2}\right\rangle_{\mathrm{st}}+\Delta_{p^{2}},\langle x p\rangle=\Delta_{x p}$, and perform linear stability analysis in $\Delta$ 's:

$$
\begin{aligned}
\partial_{t}\left(\Delta_{x^{2}}\right)= & \frac{2}{m} \Delta_{x p}, \\
\partial_{t}\left(\Delta_{x p}\right)= & \frac{\Delta_{p^{2}}}{m}-m \Omega^{2} \Delta_{x^{2}}-\frac{24 C_{x p}\left\langle x^{2}\right\rangle_{\mathrm{st}} \Delta_{x p}+8 \hbar D_{x p} \Delta_{x^{2}}}{m \Omega a^{2}} \\
& -\frac{4 C_{p p}\left(\left\langle x^{2}\right\rangle_{\mathrm{st}} \Delta_{p^{2}}+\left\langle p^{2}\right\rangle_{\mathrm{st}} \Delta_{x^{2}}\right)+8 \hbar D_{p p} \Delta_{x p}}{m^{2} \Omega^{2} a^{2}}, \\
\partial_{t}\left(\Delta_{p^{2}}\right)= & -2 m \Omega^{2} \Delta_{x p}-\frac{16 C_{x p}}{m \Omega a^{2}}\left[\left\langle p^{2}\right\rangle_{\mathrm{st}} \Delta_{x^{2}}+\left\langle x^{2}\right\rangle_{\mathrm{st}} \Delta_{p^{2}}\right] \\
& -\frac{24 C_{p p}}{m^{2} \Omega^{2} a^{2}}\left\langle p^{2}\right\rangle_{\mathrm{st}} \Delta_{x p}+\frac{8 \hbar D_{x x}}{a^{2}} \Delta_{x^{2}}-\frac{8 \hbar D_{p p}}{m^{2} \Omega^{2} a^{2}} \Delta_{p^{2}} .
\end{aligned}
$$

The stability requires that the real parts of all eigenvalues of the matrix governing the above linear evolution have to be negative, i.e., have to describe damping. Numerical analysis of the eigenvalues of this matrix is presented in Fig. 5(c). The plot indicates that all eigenvalues are negative in most of the region of existence of the physically sound Gaussian stationary solution, but at the same time that the region of validity rapidly shrinks with increasing damping $\Gamma$. To resume, regions colored in green are not accessible by the system because either the normalized standard deviations $\delta_{x}^{2}$ and $\delta_{p}^{2}$ have an unphysical imaginary part, or they do not satisfy the Heisenberg bound $\delta_{x}^{2} \delta_{p}^{2} \geqslant 1$, or the equations for the first moments do not describe a damped harmonic oscillator [i.e., inequalities in Eq. (63) are not satisfied], or at least one of the eigenvalues of the linear stability matrix of the second moments (65) becomes positive.

Note that besides the stability question, Eqs. (64) and (65) incorporate quantum dynamical effects: they describe dynamics clearly different from their high- $T$ classical analogs, due to the quantum form and origin of the diffusion coefficients $D_{x x}, D_{x p}$, and $D_{p p}$.

Finally, let us comment about the large prohibited region we find in the quadratic case at large $T$. This region is generally dynamically unstable, and arises because of the diverging fluctuations in $x$ caused by a large Lamb shift of the effective trap frequency, which turns the attractive harmonic potential into an effectively repulsive one. It is reasonable to expect that this region would become allowed if we added a quartic term to the confinement, on top of the usual quadratic one. Indeed, $\mathrm{Hu}, \mathrm{Paz}$, and Zhang considered only this case, for nonlinear couplings [8]. However, traps for ultracold atoms are generally to a very high approximation purely quadratic in the region 
where the atoms are confined, so that the presence of a quartic component may be unjustified in a real experiment.

\section{CONCLUSIONS}

We have presented in this paper a careful discussion of quantum Brownian motion in the case when the reservoir exhibits an energy cutoff $\hbar \Lambda$ much larger than other energy scales. We considered a Brownian particle in a harmonic trap, and derived and discussed validity of QME in this limit for the case of linear and various forms of nonlinear couplings to the bath. We have pointed out that stationary distributions exhibit elliptical deformations, and in the case of nonlinear coupling even genuine quantum squeezing along $x$.

An ideal application of this theory would be the study of the properties of impurity atoms embedded into a Bose-Einstein condensate or an ultracold Fermi gas. A possible detection of predicted effects would require us to (i) embed a dilute and weakly interacting gas of impurities in a degenerate ultracold gas; (ii) monitor the stationary distribution of impurities; (iii) eventually, monitor their approach toward equilibrium. The application of our theory to such situations may be implemented along the lines sketched in Appendix F.

Another interesting question concerns the SmoluchowskiKramers limit [11,12], which can be considered as a regime of overdamped quantum Brownian motion, or the case where the mass $m$ of the Brownian particle tends to zero. This limit is already highly nontrivial at the classical level, in the presence of the inhomogeneous damping and diffusion, and it requires a careful application of homogenization theory (cf. $[13,14,89,90])$. Of course, the theoretical approach here is based on the separation of time scales, and has been in other contexts studied in the theory of classical and quantum stochastic process $[87,88]$. In particular, the theory of adiabatic elimination has been developed to include the short-time non-Markovian "initial slip" effects and the effective long-time dynamics of the systems and the bath ("adiabatic drag") (cf. [76-78] and references therein).

The Smoluchowski-Kramers (SK) limit was also intensively studied in the contexts of Caldeira-Leggett model and quantum Brownian motion (cf. [91,92] and references therein). The problem with this limit is that it corresponds to strong damping, and evidently cannot be described using weak-coupling approach that is normally used to derive the QME from the microscopic model in the Born-Markov approximation. We envisage here two possible and legitimate lines of investigation.

One can forget about the microscopic derivation, and take the Born-Markov QME as a starting point. The SK limit corresponds then to setting the spring constant $m \Omega^{2}$ and friction $\eta$ to constants, and letting the mass $m \rightarrow 0$, so that $\gamma \rightarrow \infty$ as $1 / m$ and $\Omega \rightarrow \infty$ as $1 / \sqrt{m}$. The aim is to eliminate the fast variable (the momentum) and to obtain the resulting equation for the position of the Brownian particle; again, the
Wigner function formalism is particularly suited for such a task.

More ambitious and physically more sound is the approach in which the microscopic model is treated seriously, and appropriate scalings are introduced at the microscopic level. One can then start, for instance, from the formally exact path-integral expression for the reduced dynamics, as pursued by Ankerhold and collaborators [91,92]. The other possibility is to use a restricted version of the weak-coupling assumption, only demanding that the system does not influence the bath, and use Eq. (12) combined with Laplace transform techniques and Zwanzig's approach [93].

To our knowledge, neither of the two above proposed research tasks has been so far realized for the case of inhomogeneous damping and diffusion.

Last, but not least, we must bear in mind that the QMEs derived and discussed in this work suffer from the fact that they do not, in general, have the Lindblad form, and thus their solutions are not guaranteed to correspond to physically sound, non-negatively defined density matrices. One should stress that, similarly as in the case of the (in)famous sign problem in the Monte Carlo studies of many-fermion systems, these solutions still may serve very well as generators of averages and moments, as long as the negative part of the density matrix is relatively small with respect to the positive part (in any "reasonable" matrix norm). If this is not the case, or just for formal reasons, one may add artificially "minimal" terms that assure the Lindblad form of the master equation [2,3,94,95]. It would eventually be very interesting to generalize these methods to the QMEs describing inhomogeneous damping and diffusion, and to see how these terms affect the stationary solutions and dynamics discussed in this paper.

\section{ACKNOWLEDGMENTS}

Insightful discussion with A. Celi, E. Demler, M. García Parajo, C. Gardiner, J. Lapeyre, C. Manzo, P. Szańkowski, M. Trippenbach, and G. Volpe are gratefully acknowledged. This research has been supported through ERC Advanced Grant OSYRIS (led by M.L.), EU IP SIQS, EU STREP EQuaM, John Templeton Foundation, and Spanish Ministry Project FOQUS. P.M. acknowledges funding from a Spanish MINECO "Ramón y Cajal" fellowship.

\section{APPENDIX A: MARKOVIAN QME FOR GENERIC COUPLING}

We consider here an interaction term with a completely general coupling in the position of the particle:

$$
H_{\mathrm{int}}=\sum_{k} \kappa_{k} \sqrt{\frac{\hbar}{2 m_{k} \omega_{k}}} f(x)\left(g_{k}^{\dagger}+g_{k}\right)
$$

If $f \in \mathcal{C}^{\infty}(I)$ and thus may be expanded in Taylor series, the master equation can be written in the form

$$
\dot{\rho}=-i\left[H_{S}, \rho\right]-\sum_{j, n=0}^{\infty} \sum_{k=0}^{n} \frac{\tilde{f}^{(j)} \tilde{f}^{(n)}}{a^{j+n-2} j ! n !(m \Omega)^{k}}\left\{x^{j}, \frac{i C_{n, k}}{\hbar}\left\{\sigma\left(x^{n-k} p^{k}\right), \rho\right\}+\frac{D_{n, k}}{\hbar}\left[\sigma\left(x^{n-k} p^{k}\right), \rho\right]\right\}
$$


where $\sigma\left(x^{m} p^{k}\right)$ is the sum of the $\frac{(m+k) !}{m ! k !}$ distinguishable permutations of the $m+k$ operators in the polynomial $x^{m} p^{k}$ [e.g., $\left.\sigma\left(x^{2} p\right)=x^{2} p+x p x+p x^{2}\right]$. In analogy with the preceding sections, we have introduced here

$$
\begin{aligned}
& C_{n, k}(\Omega)=(-1)^{k+1} \int_{0}^{\infty} d \tau \eta(\tau) \cos ^{n-k}(\xi) \sin ^{k}(\xi), \\
& D_{n, k}(\Omega)=(-1)^{k} \int_{0}^{\infty} d \tau \nu(\tau) \cos ^{n-k}(\xi) \sin ^{k}(\xi),
\end{aligned}
$$

where $\xi=\Omega \tau$. These integrals may be calculated by Laplace transformation, as detailed in Appendix B. Alternatively, we will outline in Appendix $\mathrm{C}$ a simpler method which employs standard trigonometric identities to straightforwardly reduce every $C_{n, k}$ to a linear combination of $C_{x}$ and $C_{p}$ (the ones computed in the linear case), and similarly every $D_{n, k}$ in terms of $D_{x}$ and $D_{p}$. As an example, since $\cos ^{3}(\xi) \sin (\xi)=[2 \sin (2 \xi)+\sin (4 \xi)] / 8$, it is obvious that $D_{4,1}(\Omega)=\left[2 D_{p}(2 \Omega)+D_{p}(4 \Omega)\right] / 8$.

In complete analogy with the linear and quadratic cases, for a power-law coupling with $f(x)=a(x / a)^{n}$ the coefficient $D_{n, 0}$ determines the decoherence in the position basis, which for a quantum superposition of two states centered, respectively, at $x$ and $x^{\prime}$ happens with a characteristic rate $\gamma_{x_{1}, x_{2}}^{(n)}=$ $D_{n, 0}\left(x_{1}^{n}-x_{2}^{n}\right)^{2} / \hbar a^{2 n-2}$ (see Fig. 2). As a consequence, for an even more general coupling containing various powers of $(x / a)$, the total decay rate in position space reads as

$$
\gamma_{x_{1}, x_{2}}=\sum_{j, n=0}^{\infty} \frac{\tilde{f}^{(j)} \tilde{f}^{(n)} D_{n, 0}\left(x_{1}^{n}-x_{2}^{n}\right)^{2}}{\hbar j ! n ! a^{j+n-2}} .
$$

In contrast with Ref. [8], we find here that quantum superpositions which are sharply localized at positions symmetric with respect to the origin (e.g., in the vicinity of, say, $x_{0}$ and $-x_{0}$ ) will be characterized by a vanishing decoherence rate (i.e., a diverging lifetime) in presence of couplings which contain only even powers of $n$.

\section{Large-cutoff limit (general case)}

In the limit $\Lambda \gg T, \Omega$, we find the following:

(i) $C_{n, k} \propto \Lambda^{1-k}$, such that at every order $n$ the only divergent term is linear, and it is the one which may be reabsorbed in the Hamiltonian; indeed, $C_{n, 0}$ is the coefficient in front of the term $i\left[x^{n},\left\{x^{n}, \rho\right\}\right]=i\left[x^{2 n}, \rho\right]$, so that the divergent term is canceled by taking $H_{\text {sys }}=H_{S}-C_{n, 0} f(x)^{2}$. Moreover, for every $n$ we have $C_{n, 1}=m \gamma \Omega / 2$.

(ii) Between the coefficients $D_{n, k}$, only the term with $k=1$ diverges, logarithmically as $D_{n, 1} \sim \frac{m \gamma \Omega}{\pi} \ln \left(\frac{\hbar \Lambda}{2 \pi k_{B} T}\right)+\cdots$. All terms with $k \neq 1$ are instead finite.

\section{High-temperature limit (general case)}

In the high-temperature limit $k_{B} T \gg \Lambda \gg \Omega$, the coefficients $C$ are as in the large-cutoff limit, as they do not depend on $T$. In the set of $D$ coefficients, only $D_{n, 0} \sim m \gamma k_{B} T / \hbar$ remains finite, while all others go to zero. Using the identity $\sigma\left(x^{n-1} p\right)=n\left\{x^{n-1}, p\right\} / 2$, it is easy to show that the master equation (A2) reduces to (21) at high temperatures. In this classical limit, we see that in presence of a nonlinear coupling, the coefficients of the QME satisfy a generalized fluctuationdissipation theorem since for any $n$ we have $D_{n, 0} / C_{n, 1} \approx$ $2 k_{B} T / \hbar \Omega$.

\section{APPENDIX B: LAPLACE TRANSFORMS}

Here, we show how to compute the coefficients of the QME with a generic coupling by direct Laplace transform. We have

$$
\begin{gathered}
C_{n, k}(\Omega)=(-1)^{k+1} \frac{m \gamma \Lambda^{2}}{2} \mathcal{L}\left[\cos ^{n-k}(\xi) \sin ^{k}(\xi)\right]_{\Lambda}, \\
D_{n, k}(\Omega)=\frac{m k_{B} T \gamma \Lambda^{2}}{\hbar} \sum_{p=-\infty}^{+\infty} \frac{1}{\Lambda^{2}-v_{p}^{2}}\left\{\Lambda \mathcal{L}\left[\cos ^{n-k}(\xi) \sin ^{k}(\xi)\right]_{\Lambda}\right. \\
\left.-\left|v_{p}\right| \mathcal{L}\left[\cos ^{n-k}(\xi) \sin ^{k}(\xi)\right]_{\left|v_{p}\right|}\right\},
\end{gathered}
$$

where $\mathcal{L}[a(\xi)]_{s}=\int_{0}^{\infty} d \xi a(\xi) e^{-s \xi}$ stands for the Laplace transform of $a(\xi)$ with respect to the variable $s$. Using the following identity, valid for $s>0$,

$$
\begin{aligned}
\mathcal{L} & {\left[\cos ^{(n-k)}(\xi) \sin ^{(k)}(\xi)\right]_{s} } \\
& =\sum_{l=0}^{n-k} \sum_{j=0}^{k}(-1)^{j+k} \frac{i^{k}}{2^{n}}\left(\begin{array}{c}
n-k \\
l
\end{array}\right)\left(\begin{array}{l}
k \\
j
\end{array}\right) \mathcal{L}\left[e^{i[n-2(j+l)] \xi}\right]_{s} \\
& =\sum_{l=0}^{n-k} \sum_{j=0}^{k}(-1)^{j+k} \frac{i^{k}}{2^{n}} F_{n j l}(s),
\end{aligned}
$$

with

$$
F_{n j l}(s) \equiv\left(\begin{array}{c}
n-k \\
l
\end{array}\right)\left(\begin{array}{l}
k \\
j
\end{array}\right) \frac{1}{s-i[n-2(j+l)] \Omega},
$$

one readily finds

$$
C_{n, k}=\frac{m \gamma \Lambda^{2}}{2} \sum_{l=0}^{n-k} \sum_{j=0}^{k}(-1)^{j+1} \frac{i^{k}}{2^{n}} F_{n j l}(\Lambda) .
$$

In the expression for $D_{n, k}$, the zero Matsubara-frequency term should must be treated separately, so that one obtains

$$
\begin{aligned}
D_{n, k}= & \frac{i^{k}}{2^{n}} \frac{m k_{B} T \gamma}{\hbar} \sum_{l=0}^{n-k} \sum_{j=0}^{k}(-1)^{j}\left\{\Lambda F_{n j l}(\Lambda)\right. \\
& \left.+2 \sum_{p=1}^{+\infty} \frac{\Lambda^{2}}{\Lambda^{2}-v_{p}^{2}}\left[\Lambda F_{n j l}(\Lambda)-v_{p} F_{n j l}\left(v_{p}\right)\right]\right\} .
\end{aligned}
$$

\section{APPENDIX C: TRIGONOMETRIC IDENTITIES}

The identities presented here provide a very simple method (alternative to the one described in Appendix B) to compute the $2 n+2$ coefficients needed to describe the QME for an arbitrary coupling $f(x) \propto x^{n}$ in terms of just the two integrals $I_{\nu} \equiv \int_{0}^{\infty} d \tau \nu(\tau)$ and $I_{\eta} \equiv \int_{0}^{\infty} d \tau \eta(\tau)$, and of the four coefficients $\left\{C_{x}, C_{p}, D_{x}, D_{p}\right\}$ we derived for a linear coupling. Take $p+q=n$. 
Whenever $p$ is even (or zero), we have

$$
\begin{aligned}
\sin ^{p}(x) \cos ^{q}(x) & =\left[1-\cos ^{2}(x)\right]^{p / 2} \cos ^{q}(x) \\
& =c_{0}+\sum_{k=0}^{\mathcal{F}[(n-1) / 2]} \alpha_{k} \cos [(n-2 k) x],
\end{aligned}
$$

where $\mathcal{F}(x)$ is the "floor" function (giving the greatest integer less than or equal to $x$ ), and $c_{0}$ and $\left\{\alpha_{k}\right\}$ are constants which may be determined using the power reduction trigonometric formulas [96]. As an example, we find

$$
\begin{aligned}
\sin ^{2}(x) \cos ^{3}(x)= & \frac{3 \cos (x)+\cos (3 x)}{4} \\
& -\frac{10 \cos (x)+5 \cos (3 x)+\cos (5 x)}{16} .
\end{aligned}
$$

This formula reduces high powers of the trigonometric quantity to a sum of cosine functions of multiples of its argument, thereby reducing the desired integrals to known ones.

Similarly, whenever $q$ is even (or zero), we have

$$
\begin{aligned}
\sin ^{p}(x) \cos ^{q}(x)= & \sin ^{p}(x)\left[1-\sin ^{2}(x)\right]^{q / 2} \\
& =c_{0}+\sum_{k=0}^{\mathcal{F}[(n-1) / 2]} \alpha_{k} \sin [(n-2 k) x] .
\end{aligned}
$$

In the case where both $p$ and $q$ are odd integers, we may write

$$
\begin{aligned}
& \sin ^{p}(x) \cos ^{q}(x) \\
& \quad=\sin (x) \cos (x)\left[1-\cos ^{2}(x)\right]^{\frac{p-1}{2}} \cos ^{q-1}(x) \\
& \quad=\frac{\sin (2 x)}{2}\left\{c_{0}+\sum_{k=0}^{\mathcal{F}[(n-3) / 2]} \alpha_{k} \cos [(n-2 k) x]\right\},
\end{aligned}
$$

and the resulting integrals may be computed using the simple identity, valid for $n>0$,

$$
\sin (2 x) \cos (2 n x)=\frac{\sin [(2 n+2) x]-\sin [(2 n-2) x]}{2} .
$$

\section{APPENDIX D: ASYMPTOTIC VALUES OF THE QME COEFFICIENTS FOR THE LINEAR AND QUADRATIC CASES}

We provide here a table summarizing the asymptotic values of the coefficients of the QME for an Ohmic spectral with a Lorentz-Drude cutoff, in presence of linear and quadratic couplings, and in various interesting limits. For simplicity of notation, we give here the values of the dimensionless quantities $c_{\ldots} \equiv 2 C_{\ldots} /(m \gamma \Omega)$ (and similarly for $\left.d_{\ldots}\right)$. In the central column, $\hbar \Omega$ and $k_{B} T$ are assumed to be of the same order of magnitude, and both much smaller than $\hbar \Lambda$.

The coefficients for a linear coupling read as

\begin{tabular}{lccc}
\hline \hline & $\frac{k_{B} T}{\hbar} \gg \Lambda \gg \Omega$ & $\Lambda \gg \Omega \sim \frac{k_{B} T}{\hbar}$ & $\Lambda \gg \Omega \gg \frac{k_{B} T}{\hbar}$ \\
\hline$c_{x}$ & $-\Lambda / \Omega$ & $-\Lambda / \Omega$ & $-\Lambda / \Omega$ \\
$c_{p}$ & 1 & 1 & 1 \\
$d_{x}$ & $\frac{2 k_{B} T}{\hbar \Omega}$ & $\operatorname{coth}\left(\frac{\hbar \Omega}{2 k_{B} T}\right)$ & 1 \\
$d_{p}$ & $-\frac{2 k_{B} T}{\hbar \Lambda}$ & $\frac{2}{\pi} \ln \left(\frac{\hbar \Lambda}{2 \pi k_{B} T}\right)$ & $\frac{2}{\pi} \ln \left(\frac{\Lambda}{\Omega}\right)$ \\
\hline \hline
\end{tabular}

The coefficients for a quadratic coupling instead read as

\begin{tabular}{lccc}
\hline \hline & $\frac{k_{B} T}{\hbar} \gg \Lambda \gg \Omega$ & $\Lambda \gg \Omega \sim \frac{k_{B} T}{\hbar}$ & $\Lambda \gg \Omega \gg \frac{k_{B} T}{\hbar}$ \\
\hline$c_{x x}$ & $-\Lambda / \Omega$ & $-\Lambda / \Omega$ & $-\Lambda / \Omega$ \\
$c_{x p}$ & 1 & 1 & 1 \\
$c_{p p}$ & $-2 \Omega / \Lambda$ & $-2 \Omega / \Lambda$ & $-2 \Omega / \Lambda$ \\
$d_{x x}$ & $\frac{2 k_{B} T}{\hbar \Omega}$ & $\frac{k_{B} T}{\hbar \Omega}+\operatorname{coth}\left(\frac{\hbar \Omega}{k_{B} T}\right)$ & 1 \\
$d_{x p}$ & $-\frac{2 k_{B} T}{\hbar \Lambda}$ & $\frac{2}{\pi} \ln \left(\frac{\hbar \Lambda}{2 \pi k_{B} T}\right)$ & $\frac{2}{\pi} \ln \left(\frac{\Lambda}{2 \Omega}\right)$ \\
$d_{p p}$ & $-\frac{\hbar \Omega}{3 k_{B} T}$ & $\frac{k_{B} T}{\hbar \Omega}-\operatorname{coth}\left(\frac{\hbar \Omega}{k_{B} T}\right)$ & -1 \\
\hline \hline
\end{tabular}

\section{APPENDIX E: HIGH-T LIMIT WITH LEADING QUANTUM CORRECTIONS}

Let us now apply the Wigner function formalism to the generalized ME (21) valid in the oversimplified high- $T$ limit, and obtain ${ }^{3}$

$$
\begin{aligned}
\dot{W}= & -\frac{i}{\hbar}\left[\frac{p_{-}^{2}-p_{+}^{2}}{2 m}+V\left(x_{+}\right)-V\left(x_{-}\right)\right] W \\
& -\frac{i \gamma}{4 \hbar}\left[f\left(x_{+}\right)-f\left(x_{-}\right)\right]\left(\left\{p_{-}, f^{\prime}\left(x_{+}\right)\right\}+\left\{p_{+}, f^{\prime}\left(x_{-}\right)\right\}\right) W \\
& -\frac{\gamma m k T}{\hbar^{2}}\left[f^{2}\left(x_{+}\right)+f^{2}\left(x_{-}\right)-2 f\left(x_{+}\right) f\left(x_{-}\right)\right] W . \quad(\mathrm{E} 1)
\end{aligned}
$$

In the case, when the potential $V(x)$ is nonharmonic and/or $f(x)$ is not a linear or quadratic function of $x$, to proceed further we perform a Taylor expansion in $\hbar$, and keep the leading terms only. In other words, we attempt to include the leading quantum corrections. One finds then

$$
\begin{aligned}
\dot{W}= & {\left[-\partial_{x} \frac{p}{m}+\partial_{p} V^{\prime}(x)-\frac{\hbar^{2}}{24} \partial_{p}^{3} V^{\prime \prime \prime}(x)+\cdots\right] W } \\
& +\gamma\left[\partial_{p} p\left[f^{\prime}(x)\right]^{2}+\frac{\hbar^{2} \partial_{p}^{2}}{8}\left(2 \partial_{x} f^{\prime}(x) f^{\prime \prime}(x)\right.\right. \\
& \left.\left.-2\left[f^{\prime \prime}(x)\right]^{2}-\frac{4}{3} \partial_{p} p f^{\prime}(x) f^{\prime \prime \prime}(x)\right)+\cdots\right] W \\
& +m \gamma k_{B} T\left[\partial_{p}^{2}\left[f^{\prime}(x)\right]^{2}-\frac{\hbar^{2}}{12} \partial_{p}^{4} f^{\prime}(x) f^{\prime \prime \prime}(x)+\cdots\right] W .
\end{aligned}
$$

The above equation is the main result of this section: it combines the (oversimplified) high- $T$ limit with the leading quantum corrections. To zeroth order in $\hbar$, the ME for the Wigner matrix reads as

$$
\begin{aligned}
\dot{W}= & \left\{-\frac{p}{m} \partial_{x}+V^{\prime}(x) \partial_{p}+\gamma\left[f^{\prime}(x)\right]^{2} \partial_{p} p\right. \\
& \left.+m \gamma k_{B} T\left[f^{\prime}(x)\right]^{2} \partial_{p}^{2}\right\} W .
\end{aligned}
$$

\footnotetext{
${ }^{3}$ Note $\quad$ that $\quad\{\dot{f}(\hat{x}), \rho\} f(\hat{x})=\frac{\left\{p_{-}, f^{\prime}\left(x_{+}\right)\right\}+\left\{p_{+}, f^{\prime}\left(x_{-}\right)\right\}}{2 m} \rho f(\hat{x})=$ $f\left(x_{-}\right) \frac{\left\{p_{-}, f^{\prime}\left(x_{+}\right)\right\}+\left\{p_{+}, f^{\prime}\left(x_{-}\right)\right\}}{2 m} W$.
} 


\section{Quadratic case: High-T solution}

As an example, we consider the simplest nonlinear coupling to the bath, a quadratic one, which we write in the form $f(x)=x^{2} / a$. We also take the potential to be quadratic, $V(x)=m \Omega^{2} x^{2} / 2$. Since $f^{\prime \prime \prime}(x)=0$, from Eq. (E2) truncated to $O\left(\hbar^{2}\right)$ we have

$$
\begin{aligned}
\dot{W}= & {\left[-\frac{p}{m} \partial_{x}+m \Omega^{2} x \partial_{p}\right.} \\
& \left.+\frac{4 \gamma x^{2}}{a^{2}}\left(\partial_{p} p+m k_{B} T \partial_{p}^{2}+\frac{\hbar^{2}}{4 x} \partial_{p}^{2} \partial_{x}\right)\right] W .
\end{aligned}
$$

A stationary solution of this equation is in the form of Eq. (43) with $\sigma_{p}=\sigma_{x}=1$ and

$$
\tilde{T}=\frac{T}{2}\left[1 \pm \sqrt{1-\left(\frac{\hbar \Omega}{k_{B} T}\right)^{2}}\right] .
$$

Only the + solution is physically acceptable, as can be seen by looking at large temperature $k_{B} T \gg \hbar \Omega$, where the + solution becomes

$$
\tilde{T}=T\left[1-\left(\frac{\hbar \Omega}{2 k T}\right)^{2}\right] .
$$

This result is plotted as a red curve in Fig. 3, and may be interpreted as an effective cooling since $\tilde{T}<T$, or as a breakdown of the dissipation-fluctuation relation, or as quantum localization in phase space. However, as we have seen, this result is incorrect. Obviously, it cannot be correct when $k_{B} T \simeq \hbar \Omega$, but it loses validity already at larger temperatures, when $k_{B} T \lesssim \hbar \Lambda$ since then neither $D_{x p}$ nor $D_{p p}$ terms can be neglected. Looking from another angle, this result contains a quantum correction of order $\hbar \Omega / k_{B} T$, which is simply nonsystematic, and moreover it depends on the order of limits: high temperature $T \rightarrow \infty$, and stationarity, long-time limit $t \rightarrow \infty$.

\section{APPENDIX F: HARMONICALLY TRAPPED PARTICLE INSIDE A BOSE-EINSTEIN CONDENSATE}

The problem of dilute impurities in an ultracold gas can be studied from various points of view: as a polaron problem in a Fermi (cf. [42-47]) or Bose (cf. [48-57]) gas, or as problem of orthogonality catastrophe in a Fermi gas (cf. [97,98]), or with established techniques for studying polarons in condensed matter systems [58]. We propose yet another point of view. We consider a condensate of $N \gg 1$ identical bosonic atoms of mass $M$ inside an harmonic trap of frequency $\omega$, interacting with scattering length $a_{s}$. Denoting by $\psi^{\dagger}(\mathbf{r})$ and $\hat{\psi}(\mathbf{r})$ atomic creation and annihilation operators, the Hamiltonian of the Bose-Einstein condensate (BEC) is given by

$$
\begin{aligned}
H_{\mathrm{BEC}}= & \int d^{3} \mathbf{r} \hat{\psi}^{\dagger}(\mathbf{r})\left[-\frac{\hbar^{2} \nabla^{2}}{2 M}+\frac{M \omega^{2} \mathbf{r}^{2}}{2}\right. \\
& \left.+\frac{4 \pi \hbar^{2} a_{s}}{M} \hat{\psi}^{\dagger}(\mathbf{r}) \hat{\psi}(\mathbf{r})\right] \hat{\psi}(\mathbf{r}) .
\end{aligned}
$$

We consider a single impurity trapped inside the BEC. The impurity is described as a harmonic oscillator of mass $m$ and frequency $\Omega$, interacting with the BEC atoms through a shortrange (contact) potential characterized by a scattering length $a_{s}$. Its mean-field Hamiltonian is

$$
H_{\mathrm{imp}}=-\frac{\hbar^{2} \nabla^{2}}{2 m}+\frac{m \Omega^{2} \mathbf{r}^{2}}{2}+\frac{2 \pi \hbar^{2} a_{s}}{\mu} n(\mathbf{r}),
$$

where $\mu=m M /(m+M)$ is the reduced mass, and $n(\mathbf{r})=$ $\hat{\psi}^{\dagger}(\mathbf{r}) \hat{\psi}(\mathbf{r})$ is the BEC density. We follow the Bogoliubov-de Gennes $(\mathrm{BdG})$ formalism [99], and write $\hat{\psi}(\mathbf{r})=\sqrt{N} \varphi(\mathbf{r})+$ $\delta \hat{\psi}(\mathbf{r})$ with $\varphi$ real, $\int d^{3} \mathbf{r} \varphi^{2}(\mathbf{r})=1$, and

$$
\begin{array}{r}
\delta \hat{\psi}(\mathbf{r})=\sum_{\mathbf{k}} \hat{g}_{\mathbf{k}} u_{\mathbf{k}}(\mathbf{r})+\hat{g}_{-\mathbf{k}}^{\dagger} v_{\mathbf{k}}^{*}(\mathbf{r}), \\
\delta \hat{\psi}^{\dagger}(\mathbf{r})=\sum_{\mathbf{k}} \hat{g}_{\mathbf{k}}^{\dagger} u_{\mathbf{k}}^{*}(\mathbf{r})+\hat{g}_{-\mathbf{k}} v_{\mathbf{k}}(\mathbf{r}),
\end{array}
$$

where $\hat{g}_{\mathbf{k}}^{\dagger}$ and $\hat{g}_{\mathbf{k}}$ are the Bogoliubov quasiparticles' creation and annihilation operators, while $v_{-\mathbf{k}}^{*}(\mathbf{r})$ and $u_{\mathbf{k}}(\mathbf{r})$ are the corresponding mode functions. We approximate

$$
\begin{aligned}
\hat{\psi}^{\dagger}(\mathbf{r}) \hat{\psi}(\mathbf{r}) & \simeq N \varphi^{2}(\mathbf{r})+\sqrt{N} \varphi(\mathbf{r})\left[\delta \hat{\psi}(\mathbf{r})+\delta \hat{\psi}^{\dagger}(\mathbf{r})\right] \\
& =n(\mathbf{r})+\sqrt{n(\mathbf{r})}\left[\sum_{\mathbf{k}} \hat{g}_{\mathbf{k}} f_{\mathbf{k}}(\mathbf{r})+\hat{g}_{\mathbf{k}}^{\dagger} f_{\mathbf{k}}^{*}(\mathbf{r})\right]
\end{aligned}
$$

with $f_{\mathbf{k}}(\mathbf{r})=u_{\mathbf{k}}(\mathbf{r})+v_{-\mathbf{k}}(\mathbf{r})$. As the phases of $u$ and $v$ are arbitrary, we may choose them real, such that $f_{\mathbf{k}}(\mathbf{r})=f_{\mathbf{k}}^{*}(\mathbf{r})$. The BdG Hamiltonian for the impurity + BEC becomes then

$$
\begin{aligned}
H_{\mathrm{BdG}}= & -\frac{\hbar^{2} \nabla^{2}}{2 m}+\frac{m \Omega^{2} \mathbf{r}^{2}}{2}+\sum_{\mathbf{k}} \hbar \omega_{\mathbf{k}} \hat{g}_{\mathbf{k}}^{\dagger} \hat{g}_{\mathbf{k}} \\
& +\frac{2 \pi \hbar^{2} a_{s}}{\mu}\left[n(\mathbf{r})+\sqrt{n(\mathbf{r})} \sum_{\mathbf{k}} f_{\mathbf{k}}(\mathbf{r})\left(\hat{g}_{\mathbf{k}}+\hat{g}_{\mathbf{k}}^{\dagger}\right)\right] .
\end{aligned}
$$

There are several important differences between the $\mathrm{BdG}$ model (F4) and the Caldeira-Leggett model:

(i) In the CLM, the interaction Hamiltonian has a simple separable form $H_{I}=-\hat{B} f(\hat{x})$, where $\hat{B}$ and $f(\hat{x})$ are bath and system operators, respectively. This is not the case in the BdG model: different Bogoliubov modes couple differently to the system via different mode functions.

(ii) The spectral density for a BEC is not necessarily Ohmic. It depends on the dimension, and the dispersion relation of the Bogoliubov modes $\hbar \omega_{\mathbf{k}}$; this relation generally interpolates between low-energy phononlike $\left(\hbar \omega_{\mathbf{k}} \propto|\mathbf{k}|\right)$ and high-energy free-particle-like ( $\left.\hbar \omega_{\mathbf{k}} \propto \mathbf{k}^{2}\right)$ behaviors (cf. [99]), and it may even exhibit a roton minimum at intermediate energies (cf. [100]).

(iii) In any practical physical application of the present theory, the cutoff energy $\hbar \Lambda$ has a very concrete physical sense: in a trap the bath frequencies are evidently bound by the trap depth, in an optical lattice by the lowest band's width, and so on. Even more seriously: In any tight trap, the high-energy excitation modes will be concentrated at the semiclassical edges, as determined by the trap potential at a given energy; their overlap with the condensate, which has a size limited, say, by the Thomas-Fermi radius, will then be very small, and will decrease rapidly with the energy of excitations. 
Radically different is the case of a Fermi bath. In this case there is no condensate, so the density fluctuations are from the very beginning quadratic functions of the fermionic creation and annihilation operators. Still, a theory similar to the one presented here may be used in situations where bosonization theory works $[101,102]$, i.e., typically in specific
1D systems. There are rare examples of Fermi surfaces for which bosonization, or in this case better to say LuttingerTomonaga theory, works [103]. If we cannot use bosonization theory, the Fermi bath has to be treated according to its fermionic identity. These problems lead, however, far beyond the scope of this paper.
[1] C. Gardiner and P. Zoller, Quantum Noise: A Handbook of Markovian and Non-Markovian Quantum Stochastic Methods with Applications to Quantum Optics, Springer Series in Synergetics (Springer, Berlin, 2004).

[2] H. Breuer and F. Petruccione, The Theory of Open Quantum Systems (Oxford University Press, Oxford, 2007).

[3] M. Schlosshauer, Decoherence and the Quantum-To-Classical Transition, The Frontiers Collection (Springer, Berlin, 2007).

[4] W. v. Waldenfels, A Measure Theoretical Approach to Quantum Stochastic Processes, Lecture Notes in Physics (Springer, Heidelberg, 2014).

[5] U. Weiss, Quantum Dissipative Systems (World Scientific, Singapore, 2008).

[6] R. Landauer, Spatial Variation of Currents and Fields Due to Localized Scatterers in Metallic Conduction, IBM J. Res. Dev. 149, 223 (1957).

[7] M. I. Dykman and M. A. Krivoglaz, Spectral distribution of nonlinear oscillators with nonlinear friction due to a medium, Phys. Status Solidi B 68, 111 (1975).

[8] B. L. Hu, J. P. Paz, and Y. Zhang, Quantum Brownian motion in a general environment. II. Nonlinear coupling and perturbative approach, Phys. Rev. D 47, 1576 (1993).

[9] T. A. Brun, Quasiclassical equations of motion for nonlinear Brownian systems, Phys. Rev. D 47, 3383 (1993).

[10] S. Banerjee and R. Ghosh, General quantum Brownian motion with initially correlated and nonlinearly coupled environment, Phys. Rev. E 67, 056120 (2003).

[11] M. V. Smoluchowski, Drei Vorträge über Diffusion, Brownsche Bewegung und Koagulation von Kolloidteilchen, Physik. Zeitschr. 17, 557 (part I) (1916); 17, 585 (part II) (1916).

[12] H. Kramers, Brownian motion in a field of force and the diffusion model of chemical reactions, Physica (Amsterdam) 7, 284 (1940).

[13] S. Hottovy, G. Volpe, and J. Wehr, Noise-induced drift in stochastic differential equations with arbitrary friction and diffusion in the Smoluchowski-Kramers limit, J. Stat. Phys. 146, 762 (2012).

[14] S. Hottovy, A. McDaniel, G. Volpe, and J. Wehr, The Smoluchowski-Kramers limit of stochastic differential equations with arbitrary state-dependent friction, Commun. Math. Phys. 336, 1259 (2015).

[15] A. McDaniel, O. Duman, G. Volpe, and J. Wehr, An SDE approximation for stochastic differential delay equations with colored state-dependent noise, arXiv:1406.7287 (2015) [Markov Processes and Related Fields (to be published)].

[16] G. Volpe, L. Helden, T. Brettschneider, J. Wehr, and C. Bechinger, Influence of Noise on Force Measurements, Phys. Rev. Lett. 104, 170602 (2010).

[17] T. Brettschneider, G. Volpe, L. Helden, J. Wehr, and C. Bechinger, Force measurement in the presence of Brownian noise: Equilibrium-distribution method versus drift method, Phys. Rev. E 83, 041113 (2011).

[18] G. Pesce, A. McDaniel, S. Hottovy, J. Wehr, and G. Volpe, Stratonovich-to-Itô transition in noisy systems with multiplicative feedback, Nature Communications 4, 2733 (2013).

[19] S. Hottovy, G. Volpe, and J. Wehr, Thermophoresis of Brownian particles driven by coloured noise, Europhys. Lett. 99, 60002 (2012).

[20] J. W. Haus and K. Kehr, Diffusion in regular and disordered lattices, Phys. Rep. 150, 263 (1987).

[21] S. Havlin and D. Ben-Avraham, Diffusion in disordered media, Adv. Phys. 36, 695 (1987).

[22] J.-P. Bouchaud and A. Georges, Anomalous diffusion in disordered media: Statistical mechanisms, models and physical applications, Phys. Rep. 195, 127 (1990).

[23] J. Klafter and I. M. Sokolov, First Steps in Random Walks (Oxford University Press, Oxford, 2011).

[24] R. Metzler and J. Klafter, The restaurant at the end of the random walk: recent developments in the description of anomalous transport by fractional dynamics, J. Phys. A: Math. Gen. 37, R161 (2004).

[25] F. Höfling and T. Franosch, Anomalous transport in the crowded world of biological cells, Rep. Prog. Phys. 76, 046602 (2013).

[26] R. Metzler, J.-H. Jeon, A. G. Cherstvy, and E. Barkai, Anomalous diffusion models and their properties: nonstationarity, non-ergodicity, and ageing at the centenary of single particle tracking, Phys. Chem. Chem. Phys. 16, 24128 (2014).

[27] E. W. Montroll and G. H. Weiss, Random Walks on Lattices. II, J. Math. Phys. 6, 167 (1965).

[28] H. Scher and E. W. Montroll, Anomalous transit-time dispersion in amorphous solids, Phys. Rev. B 12, 2455 (1975).

[29] M. J. Saxton, Lateral diffusion in an archipelago. Singleparticle diffusion, Biophys. J. 64, 1766 (1993); Single-particle tracking: the distribution of diffusion coefficients, 72, 1744 (1997).

[30] A. G. Cherstvy and R. Metzler, Population splitting, trapping, and non-ergodicity in heterogeneous diffusion processes, Phys. Chem. Chem. Phys. 15, 20220 (2013); A. G. Cherstvy, A. V. Chechkin, and R. Metzler, Particle invasion, survival, and non-ergodicity in 2D diffusion processes with space-dependent diffusivity, Soft Matter 10, 1591 (2014).

[31] I. M. Tolić-Nørrelykke, E.-L. Munteanu, G. Thon, L. Oddershede, and K. Berg-Sørensen, Anomalous Diffusion in Living Yeast Cells, Phys. Rev. Lett. 93, 078102 (2004).

[32] I. Golding and E. C. Cox, Physical Nature of Bacterial Cytoplasm, Phys. Rev. Lett. 96, 098102 (2006).

[33] J.-H. Jeon, V. Tejedor, S. Burov, E. Barkai, C. Selhuber-Unkel, K. Berg-Sørensen, L. Oddershede, and R. Metzler, In Vivo 
Anomalous Diffusion and Weak Ergodicity Breaking of Lipid Granules, Phys. Rev. Lett. 106, 048103 (2011).

[34] A. V. Weigel, B. Simon, M. M. Tamkun, and D. Krapf, Ergodic and nonergodic processes coexist in the plasma membrane as observed by single-molecule tracking, Proc. Natl. Acad. Sci. USA 108, 6438 (2011).

[35] A. Kusumi, T. K. Fujiwara, R. Chadda, M. Xie, T. A. Tsunoyama, Z. Kalay, R. S. Kasai, and K. G. Suzuki, Dynamic Organizing Principles of the Plasma Membrane that Regulate Signal Transduction: Commemorating the Fortieth Anniversary of Singer and Nicolson's Fluid-Mosaic Model, Annu. Rev. Cell Dev. Biol. 28, 215 (2012).

[36] G. J. Bakker, C. Eich, J. A. Torreno-Pina, R. Diez-Ahedo, G. Perez-Samper, T. S. van Zanten, C. G. Figdor, A. Cambi, and M. F. García-Parajo, Lateral mobility of individual integrin nanoclusters orchestrates the onset for leukocyte adhesion, Proc. Natl. Acad. Sci. USA 109, 4869 (2012).

[37] I. I. Cisse, I. Izeddin, S. Z. Causse, L. Boudarene, A. Senecal, L. Muresan, C. Dugast-Darzacq, B. Hajj, M. Dahan, and X. Darzacq, Real-Time Dynamics of RNA Polymerase II Clustering in Live Human Cells, Science 341, 664 (2013).

[38] P. Massignan, C. Manzo, J. A. Torreno-Pina, M. F. GarcíaParajo, M. Lewenstein, and G. J. Lapeyre, Nonergodic Subdiffusion from Brownian Motion in an Inhomogeneous Medium, Phys. Rev. Lett. 112, 150603 (2014).

[39] C. Manzo, J. A. Torreno-Pina, P. Massignan, G. J. Lapeyre, Jr., M. Lewenstein, and M. F. García-Parajo, Weak ergodicity breaking of receptor motion in living cells stemming from random diffusivity, Phys. Rev. X 5, 011021 (2015).

[40] M. Lewenstein, A. Sanpera, and V. Ahufinger, Ultracold atoms in optical lattices: Simulating quantum many-body systems (Oxford University Press, Oxford, 2012).

[41] S. Krinner, D. Stadler, J. Meineke, J.-P. Brantut, and T. Esslinger, Direct Observation of Fragmentation in a Disordered, Strongly Interacting Fermi Gas, arXiv:1311.5174.

[42] A. Schirotzek, C.-H. Wu, A. Sommer, and M. W. Zwierlein, Observation of Fermi Polarons in a Tunable Fermi Liquid of Ultracold Atoms, Phys. Rev. Lett. 102, 230402 (2009).

[43] C. Kohstall, M. Zaccanti, M. Jag, A. Trenkwalder, P. Massignan, G. M. Bruun, F. Schreck, and R. Grimm, Metastability and coherence of repulsive polarons in a strongly interacting Fermi mixture, Nature (London) 485, 615 (2012).

[44] M. Koschorreck, D. Pertot, E. Vogt, B. Fröhlich, M. Feld, and M. Köhl, Attractive and repulsive Fermi polarons in two dimensions, Nature (London) 485, 619 (2012).

[45] P. Massignan, M. Zaccanti, and G. M. Bruun, Polarons, dressed molecules and itinerant ferromagnetism in ultracold Fermi gases, Rep. Prog. Phys. 77, 034401 (2014).

[46] Z. Lan and C. Lobo, A single impurity in an ideal atomic Fermi gas: current understanding and some open problems, J. Indian I. Sci. 94, 179 (2014).

[47] J. Levinsen and M. M. Parish, Strongly interacting twodimensional Fermi gases, Annual Review of Cold Atoms and Molecules Vol. 3 (Singapore, 2015), Chap 1, arXiv:1408.2737.

[48] R. Côté, V. Kharchenko, and M. D. Lukin, Mesoscopic Molecular Ions in Bose-Einstein Condensates, Phys. Rev. Lett. 89, 093001 (2002).

[49] P. Massignan, C. J. Pethick, and H. Smith, Static properties of positive ions in atomic Bose-Einstein condensates, Phys. Rev. A 71, 023606 (2005).
[50] F. M. Cucchietti and E. Timmermans, Strong-Coupling Polarons in Dilute Gas Bose-Einstein Condensates, Phys. Rev. Lett. 96, 210401 (2006).

[51] S. Palzer, C. Zipkes, C. Sias, and M. Köhl, Quantum Transport through a Tonks-Girardeau Gas, Phys. Rev. Lett. 103, 150601 (2009).

[52] J. Catani, G. Lamporesi, D. Naik, M. Gring, M. Inguscio, F. Minardi, A. Kantian, and T. Giamarchi, Quantum dynamics of impurities in a one-dimensional Bose gas, Phys. Rev. A 85, 023623 (2012).

[53] S. P. Rath and R. Schmidt, Field-theoretical study of the Bose polaron, Phys. Rev. A 88, 053632 (2013).

[54] T. Fukuhara, A. Kantian, M. Endres, M. Cheneau, P. Schauß, S. Hild, D. Bellem, U. Schollwöck, T. Giamarchi, C. Gross, I. Bloch, and S. Kuhr, Quantum dynamics of a mobile spin impurity, Nat. Phys. 9, 235 (2013).

[55] A. Shashi, F. Grusdt, D. A. Abanin, and E. Demler, Radiofrequency spectroscopy of polarons in ultracold Bose gases, Phys. Rev. A 89, 053617 (2014).

[56] F. Grusdt, A. Shashi, D. Abanin, and E. Demler, Bloch oscillations of bosonic lattice polarons, Phys. Rev. A 90, 063610 (2014).

[57] F. Grusdt, Y. E. Shchadilova, A. N. Rubtsov, and E. Demler, Renormalization group approach to the Fröhlich polaron model: application to impurity-BEC problem, arXiv: 1410.2203 .

[58] J. T. Devreese and A. S. Alexandrov, Fröhlich polaron and bipolaron: recent developments, Rep. Prog. Phys. 72, 066501 (2009).

[59] A. Alexandrov and J. Devreese, Advances in Polaron Physics, Springer Series in Solid-State Sciences (Springer, Berlin, 2009).

[60] E. H. Lieb and K. Yamazaki, Ground-State Energy and Effective Mass of the Polaron, Phys. Rev. 111, 728 (1958).

[61] E. H. Lieb and L. E. Thomas, Exact ground state energy of the strong-coupling polaron, Commun. Math. Phys. 183, 511 (1997).

[62] R. L. Frank, E. H. Lieb, R. Seiringer, and L. E. Thomas, Bipolaron and $\mathrm{N}$-polaron binding energies, Phys. Rev. Lett. 104, 210402 (2010).

[63] I. Anapolitanos and B. Landon, The Ground State Energy of the Multi-Polaron in the Strong Coupling Limit, Lett. Math. Phys. 103, 1347 (2013).

[64] J. M. Sancho, M. S. Miguel, and D. Dürr, Adiabatic elimination for systems of Brownian particles with nonconstant damping coefficients, J. Stat. Phys. 28, 291 (1982).

[65] M. Freidlin, Some remarks on the Smoluchowski-Kramers approximation, J. Stat. Phys. 117, 314 (2004).

[66] S. Cerrai and M. Freidlin, Small mass asymptotics for a charged particle in a magnetic field and long-time influence of small perturbations, J. Stat. Phys. 144, 101 (2011).

[67] M. Freidlin and W. Hu, Smoluchowski-Kramers approximation in the case of variable friction, J. Math. Sci. 179, 184 (2011).

[68] J. Shi, T. Chen, R. Yuan, B. Yuan, and P. Ao, Relation of a new interpretation of stochastic differential equations to Ito process, J. Stat. Phys. 148, 579 (2012).

[69] A. Caldeira and A. Leggett, Path integral approach to quantum Brownian motion, Phys. A (Amsterdam) 121, 587 (1983). 
[70] A. Caldeira and A. Leggett, Quantum tunneling in a dissipative system, Ann. Phys. (NY) 149, 374 (1983).

[71] C. Fleming and B. Hu, Non-Markovian dynamics of open quantum systems: Stochastic equations and their perturbative solutions, Ann. Phys. (NY) 327, 1238 (2012).

[72] K. Rzążewski and W. Żakowicz, Initial value problem and causality of radiating oscillator, J. Phys. A: Math. Gen. 9, 1159 (1976).

[73] M. Lewenstein and K. Rzążewski, Collective radiation and the near zone field, J. Phys. A: Math. Gen. 13, 743 (1980).

[74] A. G. Redfield, On the Theory of Relaxation Processes, IBM J. Res. Dev. 1, 19 (1957).

[75] K. Blum, Density Matrix Theory and Applications (Plenum, New York, 1981).

[76] F. Haake, Systematic adiabatic elimination for stochastic processes, Z. Phys. B: Condens. Matter 48, 31 (1982).

[77] F. Haake and M. Lewenstein, Adiabatic drag and initial slip in random processes, Phys. Rev. A 28, 3606 (1983).

[78] F. Haake, M. Lewenstein, and R. Reibold, in Adiabatic Drag and Initial Slip for Random Processes with Slow and Fast Variables, edited by L. Accardi and W. von Waldenfels, Lecture Notes in Mathematics: Quantum Probability and Applications II (Springer, Heidelberg, 1985).

[79] H. Grabert, P. Schramm, and G.-L. Ingold, Quantum Brownian motion: The functional integral approach, Phys. Rep. 168, 115 (1988).

[80] C. Fleming, A. Roura, and B. Hu, Exact analytical solutions to the master equation of quantum Brownian motion for a general environment, Ann. Phys. (NY) 326, 1207 (2011).

[81] P. Ullersma, An exactly solvable model for Brownian motion: I. Derivation of the Langevin equation, Physica (Amsterdam) 32, 27 (1966); An exactly solvable model for Brownian motion: II. Derivation of the Fokker-Planck equation and the master equation, 32, 56 (1966); An exactly solvable model for Brownian motion: III. Motion of a heavy mass in a linear chain, 32, 74 (1966); An exactly solvable model for Brownian motion: IV. Susceptibility and Nyquist's theorem, 32, 90 (1966).

[82] F. Haake and R. Reibold, Strong damping and low-temperature anomalies for the harmonic oscillator, Phys. Rev. A 29, 3208 (1984).

[83] W. H. Zurek, Decoherence, einselection, and the quantum origins of the classical, Rev. Mod. Phys. 75, 715 (2003).

[84] M. Schlosshauer, Decoherence, the measurement problem, and interpretations of quantum mechanics, Rev. Mod. Phys. 76, 1267 (2005).

[85] C. H. Fleming and N. I. Cummings, Accuracy of perturbative master equations, Phys. Rev. E 83, 031117 (2011).

[86] Y. Subaşi, C. H. Fleming, J. M. Taylor, and B. L. Hu, Equilibrium states of open quantum systems in the strong coupling regime, Phys. Rev. E 86, 061132 (2012).
[87] C. Gardiner, Stochastic Methods: A Handbook for the Natural and Social Sciences, Springer Series in Synergetics, Vol. 13 (Springer, Heidelberg, 2009).

[88] H. Risken, The Fokker-Planck Equation: Methods of Solution and Applications, Springer Series in Synergetics, Vol. 18 (Springer, Heidelberg, 2012).

[89] G. Papanicolaou, Modern modeling of continuum phenomena (Ninth Summer Sem. Appl. Math., Rensselaer Polytech. Inst., Troy, N.Y., 1975), Lectures in Applied Mathematics, Vol. 16 (AMS, Providence, 1977), pp. 109-147.

[90] G. Pavliotis and A. Stuart, Quantum Brownian Motion: The Functional Integral Approach, Texts in Applied Mathematics, Vol. 53 (Springer, New York, 2008).

[91] S. A. Maier and J. Ankerhold, Quantum Smoluchowski equation: A systematic study, Phys. Rev. E 81, 021107 (2010).

[92] J. Ankerhold, P. Pechukas, and H. Grabert, Strong Friction Limit in Quantum Mechanics: The Quantum Smoluchowski Equation, Phys. Rev. Lett. 87, 086802 (2001); J. Ankerhold and H. Grabert, Erratum: Strong Friction Limit in Quantum Mechanics: The Quantum Smoluchowski Equation [Phys. Rev. Lett. 87, 086802 (2001)], ibid. 101, 119903 (2008).

[93] R. Zwanzig, Memory Effects in Irreversible Thermodynamics, Phys. Rev. 124, 983 (1961).

[94] S. Gao, Dissipative Quantum Dynamics with a Lindblad Functional, Phys. Rev. Lett. 79, 3101 (1997).

[95] L. Diósi, On High-Temperature Markovian Equation for Quantum Brownian Motion, Europhys. Lett. 22, 1 (1993).

[96] I. S. Gradshteyn and I. M. Ryzhik, Table of Integrals, Series, and Products, 6th ed. (Academic, San Diego, 2000).

[97] M. Knap, A. Shashi, Y. Nishida, A. Imambekov, D. A. Abanin, and E. Demler, Time dependent impurity in ultracold fermions: orthogonality catastrophe and beyond, Phys. Rev. X 2, 041020 (2012).

[98] H. Kim and D. A. Huse, Superdiffusive nonequilibrium motion of an impurity in a Fermi sea, Phys. Rev. A 85, 043603 (2012).

[99] L. Pitaevskii and S. Stringari, Bose-Einstein Condensation (Oxford University Press, Oxford, 2003).

[100] T. Lahaye, C. Menotti, L. Santos, M. Lewenstein, and T. Pfau, The physics of dipolar bosonic quantum gases, Rep. Prog. Phys. 72, 126401 (2009).

[101] T. Giamarchi, Quantum Physics in One Dimension (Oxford University Press, Oxford, 2004).

[102] A. O. Gogolin, A. A. Nersesyan, and A. M. Tsvelik, Bosonization and Strongly Correlated Systems (Cambridge University Press, Cambridge, 2004).

[103] G. D. Mahan, Many-Particle Physics (Plenum, New York, 1993). 